\title{
ON THE EXISTENCE OF THE UCP-BASIS IN COMPLEX SEPARABLE INFINITE DIMENSIONAL BANACH SPACES
}

\author{
Nota del m.e. PAOLO TERENZI (*)
}

(Adunanza del 24 novembre 2011)

SUNTO. - Estensione al caso complesso dei sistemi biortogonali generanti e discussione della possibile estensione della UCP-base al caso complesso.

$$
* * *
$$

ABSTRACT. - Extension to the complex case of the generating biorthogonal systems and discussion on the possible extsnsion of the UCP-basis to the complex case.

\section{INTRODUCTION}

In [2] (see also [3] for a better proof), by means of a direct construction, I proved the existence of the UCP-basis in every separable infinite dimensional real Banach Space.

(*) Dipartimento di Matematica del Politecnico di Milano Piazza Leonardo Da Vinci 32-20133-Milano-Italy. E-mail: paolo.terenzi@polimi.it 
Aim of this paper is to sum up the situation for what concerns the possible existence of the UCP-Basis also in every infinite dimensional separable complex Banach space.

Let us recall the necessary definitions:

A real Banach space $X$ is a real linear space endowed with a norm $\|.$.$\| , which is a real non negative function on X$ which imitates the "absolute value" for the real numbers, hence such that, for any $x \in X$ and $y \in X,\|x\|=0$ only if $x=O$ (the null vector), $\|x+y\| \leq\|x\|+\|y\|$ and $\| \lambda x||=|\lambda| .|| x||$ for any real number $\lambda$; in particular in this topology $X$ is "complete" (in the sense that every "Cauchy sequence" $\left(x_{n}\right)_{n=1}^{\infty}$ of $X$ (for each $\varepsilon>0$ there exists a positive integer $n_{\varepsilon}$ so that $\left\|x_{n}-x_{m}\right\|<\varepsilon$ for each $n \geq n_{\varepsilon}$ and $m \geq n_{\varepsilon}$ ) converges to an element of $X$ ). If the numbers $\lambda$ of above can be also complex, we have a complex Banach space.

Let now $\left(x_{n}, x_{n}^{*}\right)_{n=1}^{\infty}$ be a biorthogonal system of a (real or complex) Banach space $X$ (that is $\left(x_{n}\right)_{n=1}^{\infty} \subset X$ and $\left(x_{n}^{*}\right)_{n=1}^{\infty} \subset X^{*}$-the dual space- with $x_{m}^{*}\left(x_{n}\right)=\delta_{m, n}$ Kronecker indices for each $m$ and $n$ ), we say that $\left(x_{n}\right)_{n=1}^{\infty}$ is a:

(i) basis with fixed brackets of $X$ if there exists an increasing sequence $(p(m))_{m=1}^{\infty}$ of positive integers such that, setting $p(0)=0$, we have the following representation for each $\bar{x}$ of $X$,

$$
\bar{x}=\sum_{m=0}^{\infty}\left(\sum_{n=p(m)+1}^{p(m+1)} x_{n}^{*}(\bar{x}) x_{n}\right),
$$

if $p(m)=m$ for each $m$, we have the Schauder basis of $\mathrm{X}$;

(ii) basis with uniformly controlled permutations (UCP-basis) of $X$ if there exists an increasing sequence $(p(m))_{m=1}^{\infty}$ of positive integers such that, for each $\bar{x}$ of $X$, there exists a permutation $(\bar{\pi}(n))_{n=1}^{\infty}$ of $(n)_{n=1}^{\infty}$ (which depends on $\bar{x}$ ) so that

$$
\bar{x}=\sum_{n=1}^{\infty} x_{\bar{\pi}(n)}^{*}(\bar{x}) x_{\bar{\pi}(n)} ;
$$

in particular these permutations cannot be quite arbitrary, because it is also

$$
(n)_{n=1}^{m} \subseteq(\bar{\pi}(n))_{n=1}^{p(m)} \subseteq(n)_{n=1}^{p(m+1)} \text { for each } m .
$$


We point out that the basis with fixed brackets (hence also the Schauder basis) in general does not exist ([1], Enflo 1973); hence the best kind of basis at our disposal in real case is just the UCP-basis; whereas the question of the existence of the UCP-basis also in complex case is still open. Therefore in section 2 we will extend to the complex case the "generating biorthogonal systems" which are, for the construction of UCP-basis, the most important key which allows the approximations of the element $\bar{x}$ by means of finite subsums of the series $\sum_{n=1}^{\infty} x_{n}^{*}(\bar{x}) x_{n}$.

Section 3 concers the general situation of the extension of the UCPbasis to the complex case, from the point of view of both the direct extension and the passage through the real associated space.

\section{THE ASSOCIATED GENERATING BIORTHOGONAL SYSTEM}

\subsection{The associated generating biorthogonal system in the real case}

Given a biorthogonal system $\left(u_{n}, u_{n}^{*}\right)_{n=1}^{P}$ of $X$, we call generating biorthogonal system associated to $\left(u_{n}, u_{n}^{*}\right)_{n=1}^{P}$ another biorthogonal system $\left(w_{n}, w_{n}^{*}\right)_{n=1}^{P}$ with all the properties which appear in the following theorem (for the proof see [2] p.22-34), which provides the procedure of the passage to the generating form in the real case.

Theorem 1 (GENERATING BIORTHOGONAL SYSTEM THEOREM (GBST))

Let $\left(u_{n}, u_{n}^{*}\right)_{n=1}^{P}$ be biorthogonal in $X$ with

$$
\left\|u_{n}\right\|=1 \text { and }\left\|u_{n}^{*}\right\|<K \text { for } 1 \leq n \leq P
$$

(bence $K>1$ ). Fix two positive integers $M^{\prime}$ and $Q$. I use, for each positive number $a$ and for each positive integers $q$ and $m$, the following notations:

$$
\begin{aligned}
E X[a, 1, q] & =a^{Q . q}, E X[a, m+1, q] \\
& =E X[a, 1, E X[a, m, q]]=a^{Q . E X[a, m, q]} .
\end{aligned}
$$

Then let $\left(w_{n}, w_{n}^{*}\right)_{n=1}^{P}$ be biorthogonal with $w_{1}=u_{1}$ and, for each $n$ with 
$2 \leq n \leq P$,

$$
\begin{gathered}
w_{n}=\sum_{k=1}^{n} \frac{u_{k}}{A_{n, k}}, A_{n, 1}=1 \text { and, for each } k \text { with } 2 \leq k \leq n, \\
A_{n, k}=E X\left[2 K, 2^{2 P^{2}(P-n+1)}+2(k-2), P\right]
\end{gathered}
$$

(in particular

$$
\begin{gathered}
A_{2,2}=E X\left[2 K, 2^{2 P^{2}(P-1)}, P\right], \\
\left.A_{P, k}=E X\left[2 K, 2^{2 P^{2}}+2(k-2), P\right]\right) .
\end{gathered}
$$

Fix $\bar{x} \in X$ with $\|\bar{x}\|=1$ and $\bar{n}$ with $1 \leq \bar{n} \leq P-1$ such that

$$
\left|u_{\bar{n}+1}^{*}(\bar{x})\right|>\frac{1}{2^{M P}} .
$$

Then for $Q$ sufficiently large (bence we precise that $Q$ depends also on $M^{\prime}$ ) there exists a sequence $(g(n))_{n=1}^{\bar{n}+1}$ of positive integers, with $g(\bar{n}+1) \leq P$ and $n \leq g(n)<g(n+1)$ for $1 \leq n \leq \bar{n}$, such that, if we fix $\bar{u} \in$ $\operatorname{span}\left(u_{n}\right)_{n=1}^{\bar{n}}$ with $\|\bar{u}\|=1$, bence

$$
\begin{array}{r}
\bar{u}=\sum_{n=1}^{\bar{n}} \bar{a}_{n} u_{n} \text { with }\left|\bar{a}_{n}\right|<K \text { for each } n \text { with } 1 \leq n \leq \bar{n}, \\
\quad \text { in particular with }\left|\bar{a}_{\widetilde{n}}\right| \geq \frac{1}{2^{M . P}} \text { and }\left|\bar{a}_{n}\right|<\frac{1}{2^{M^{\prime} . P}} \\
\quad \text { for } \widetilde{n}+1 \leq n \leq \bar{n}, \text { for some } \widetilde{n} \text { with } 1 \leq \widetilde{n} \leq \bar{n} ;
\end{array}
$$

then, setting $(f(n))_{n=1}^{\widetilde{n}}=(g(n))_{n=2}^{\widetilde{n}+1}$ if $\bar{a}_{\widetilde{n}}$ and $w_{g((\widetilde{n}+1))}^{*}(\bar{x})$ bave the same sign, otherwise $(f(n))_{n=1}^{\widetilde{n}}=(g(n))_{n=1}^{\widetilde{n}}$, there exists a sequence $\left(\bar{b}_{n}\right)_{n=1}^{\bar{n}}$ of numbers, with $\bar{b}_{n}=0$ for $\widetilde{n}+1 \leq n \leq \bar{n}$, such that

$$
\begin{array}{r}
0<\frac{\bar{b}_{n}}{w_{f(n)}^{*}(\bar{x})}<\frac{1}{2^{M . P}} \text { for } 1 \leq n \leq \bar{n}, \text { moreover } \\
\|\bar{w}-\bar{u}\|<\frac{1}{2^{M . P}} \text { where } \bar{w}=\sum_{n=1}^{\bar{n}} \bar{b}_{n} w_{f(n)} .
\end{array}
$$


In particular from the proof it easily follows that, if $\bar{u}^{\prime} \in \operatorname{span}\left(u_{n^{\prime}}\right)_{n=1}^{\bar{n}^{\prime}}$ with $\left(n^{\prime}\right)_{n=1}^{\bar{n}^{\prime}} \subset(n)_{n=1}^{\bar{n}}$ and $\left\|\bar{u}^{\prime}\right\|=1$, if $\left(g\left(n^{\prime \prime}\right)\right)_{n=1}^{\bar{n}^{\prime}+1}$ is any subsequence of $(g(n))_{n=1}^{\bar{n}+1}$ such that the sequence $\left(w_{g\left(n^{\prime \prime}\right)}^{*}(\bar{x})\right)_{n=1}^{\bar{n}^{\prime}+1}$ bas alternate signs, again there exists like above $\left(f\left(n^{\prime}\right)\right)_{n=1}^{\bar{n}^{\prime}} \subset\left(g\left(n^{\prime \prime}\right)\right)_{n=1}^{\bar{n}^{\prime}+1}$ and $\left(\bar{b}_{n}^{\prime}\right)_{n=1}^{\bar{n}^{\prime}}$ of numbers such that, for $1 \leq n \leq \bar{n}^{\prime}$,

$$
0<\frac{\bar{b}_{n}^{\prime}}{w_{f\left(n^{\prime}\right)}^{*}(\bar{x})}<\frac{1}{2^{M^{\prime} . P}},\left\|\sum_{n=1}^{\bar{n}^{\prime}} \bar{b}_{n}^{\prime} w_{f\left(n^{\prime}\right)}-\bar{u}^{\prime}\right\|<\frac{1}{2^{M^{\prime} . P}} .
$$

OBSERVATION 1. From (5) and (6) it follows that the numbers $A_{n, k}$ are strongly increasing when index $k$ increases and also more strongly increasing when index $n$ decreases; let us show a direct consequence of this fact:

(a) Let us fix $\bar{n}$ of (7) and $\bar{u}=\sum_{n=1}^{\bar{n}} \bar{a}_{n} u_{n}$ of (8) of above, suppose moreover to be going to construct $\bar{w}=\sum_{n=1}^{\bar{n}} \bar{b}_{n} w_{f(n)}$ of (9) of above, in particular fix $n$ with $1 \leq n \leq \bar{n}-1$ and suppose to be concerned with the construction of the addendum $\bar{b}_{n} w_{f(n)}$ always of (9) of above, suppose to have already precised the previous addendum

$$
\begin{gathered}
\frac{\bar{b}_{n+1}}{A_{f(n+1), n}} u_{n} \text { which appears in the development } \\
\ldots+\frac{\bar{b}_{n+1}}{A_{f(n+1), n}} u_{n}+\frac{\bar{b}_{n+1}}{A_{f(n+1), n+1}} u_{n+1}+\ldots \text { of } \bar{b}_{n+1} w_{f(n+1)}
\end{gathered}
$$

where, since $A_{f(n+1), n+1}$ is much larger than $A_{f(n+1), n}$, it follows that $\frac{\bar{b}_{n+1}}{A_{f(n+1), n}}$ is much larger than $\frac{\bar{b}_{n+1}}{A_{f(n+1), n+1}}$.

(b) Since the contribution to the construction of $\bar{a}_{n} u_{n}$ by means of the indices $f\left(n^{\prime \prime}\right)$ for $n^{\prime \prime}>n+1$ is negligible, in order to get $\bar{a}_{n} u_{n}$ we can start only from $\bar{b}_{n+1} w_{f(n+1)}$; which at its turn already gives the contribution $\frac{\bar{b}_{n+1}}{A_{f(n+1), n}} u_{n}$ to the construction of $\bar{a}_{n} u_{n}$, where we know by (a) that $\frac{\bar{b}_{n+1}}{A_{f(n+1), n}}$ is very large. 
(c) Therefore, in order to construct the addendum $\bar{b}_{n} w_{f(n)}$ of $\bar{w}=$ $\sum_{k=1}^{\bar{n}} \bar{b}_{k} w_{f(k)}$ of (9) of above, $\bar{b}_{n}$ has to be such that $\frac{\bar{b}_{n}}{A_{f(n), n}} u_{n}+$ $\frac{\bar{b}_{n+1}}{A_{f(n+1), n}} u_{n}=\bar{a}_{n} u_{n}$, therefore it follows that

$\frac{\bar{b}_{n}}{A_{f(n), n}}+\frac{\bar{b}_{n+1}}{A_{f(n+1), n}}=\bar{a}_{n},\left(\right.$ that is $\left.\bar{b}_{n}=A_{f(n), n}\left(\bar{a}_{n}-\frac{\bar{b}_{n+1}}{A_{f(n+1), n}}\right)\right)$.

(d) Since $\left|\bar{a}_{n}\right|<K$ which is a fixed number, since moreover by the end of (b) $\frac{\bar{b}_{n+1}}{A_{f(n+1), n}}$ is very large, it follows that also $\frac{\bar{b}_{n}}{A_{f(n), n}}$ is very large (moreover the sign of $\bar{b}_{n}$ has to be opposite to the sign of $\bar{b}_{n+1}$ ).

(e) Analogousy, when we pass to the construction of $\bar{a}_{n-1} u_{n-1}$, we will get that $\frac{\bar{b}_{n-1}}{A_{f(n-1), n-1}}$ will be much larger than $\frac{\bar{b}_{n}}{A_{f(n), n-1}}$, it will follow that $\frac{\bar{b}_{n-1}}{A_{f(n-1), n-1}}$ practically will "deflagrate" and so on, in a sort of "chain reaction".

In particular we notice that, also if $\left|u_{\bar{n}+1}^{*}(\bar{x})\right|>\frac{1}{2^{M P}}$ whereas $u_{n}^{*}(\bar{x})=0$ for $1 \leq n(\neq \bar{n}+1) \leq P$, the whole thesis of the theorem works; that is we can produce by means of the above procedure all the elements of $\operatorname{span}\left(u_{n}\right)_{n=1}^{\bar{n}}$ that we need.

We also notice that, by (6) and (5), if we consider $b_{n} w_{n}=$ $\sum_{k=1}^{n} \frac{b_{n}}{A_{n, k}} u_{k}$ with $\frac{\left|b_{n}\right|}{A_{n, k^{\prime}}}<\frac{1}{2^{P}}$ for some $k^{\prime}<n$, practically for our approximations we can write $b_{n} w_{n}=\sum_{k=1}^{k^{\prime}} \frac{b_{n}}{A_{n, k}} u_{m, k}$ since $\sum_{k=k^{\prime}+1}^{n} \frac{\left|b_{n}\right|}{A_{n, k}}$ becomes negligible.

For the proof of next remark see again [2] p.35-39).

Remark 2 (MODIFIED GENERATING BIORTHOGONAL SYSTEM (MGBS))

In theorem 2 we can replace $\left(w_{n}, w_{n}^{*}\right)_{n=1}^{P}$ by means of $\left(v_{n}, v_{n}^{*}\right)_{n=1}^{P}$ where, for each $n$ with $1 \leq n \leq P / 2$,

$$
\begin{gathered}
v_{2 n-1}=w_{2 n}+\frac{w_{2 n-1}}{D^{\prime}}, v_{2 n-1}^{*}=\frac{w_{2 n}^{*}}{2}+\frac{w_{2 n-1}^{*}}{2} D^{\prime}, \\
v_{2 n}=w_{2 n}-\frac{w_{2 n-1}}{D^{\prime}}, v_{2 n}^{*}=\frac{w_{2 n}^{*}}{2}-\frac{w_{2 n-1}^{*}}{2} D^{\prime},
\end{gathered}
$$


where $D^{\prime}$ is an integer $\geq 22^{\sum_{n=1}^{P}\left\|w_{n}^{*}\right\|}$. Suppose that (7), (8) and (9) of theorem 2 continue to hold. Then still there exist an integer $\widetilde{n}$ with $\bar{n} \leq$ $\widetilde{n} \leq P,(h(n))_{n=1}^{\tilde{n}}$ strictly increasing of integers and $\left(\widetilde{b}_{n}\right)_{n=1}^{\tilde{n}}$ of numbers such that again we continue to have

$$
\begin{gathered}
f(\bar{n})-1 \leq h(\widetilde{n}) \leq f(\bar{n}) \text { and } n \leq h(n)<h(n+1) \text { for each } \\
n \text { with } 1 \leq n \leq \widetilde{n}-1,0<\frac{\widetilde{b}_{n}}{v_{h(n)}^{*}(\bar{x})}<\frac{2}{2^{M^{\prime} P}} \text { for } 1 \leq n \leq \widetilde{n} \\
\quad \text { and }\|\widetilde{w}-\bar{u}\|<\frac{2}{2^{M P}} \text { where } \widetilde{w}=\sum_{n=1}^{\widetilde{n}} \widetilde{b}_{n} v_{h(n)} .
\end{gathered}
$$

OBSERVATION 2. Suppose that $(2 n-1,2 n) \subset(g(n))_{n=1}^{\bar{n}+1}$ of GBST, by the proof of GBST $\left|w_{2 n-1}^{*}(\bar{x})\right|$ is much larger than $\left|w_{2 n}^{*}(\bar{x})\right|$, moreover $D^{\prime}$ is much larger than $\left|w_{2 n-1}^{*}(\bar{x})\right|$; therefore we can approximate

$\left(v_{2 n-1}^{*}(\bar{x}) v_{2 n-1}, v_{2 n}^{*}(\bar{x}) v_{2 n}\right)$ by means of

$$
\left(\left(+\frac{D^{\prime}}{2}\right) w_{2 n-1}^{*}(\bar{x}) w_{2 n},\left(-\frac{D^{\prime}}{2}\right) w_{2 n-1}^{*}(\bar{x}) w_{2 n}\right) ;
$$

that is we have at disposal the element $w_{2 n}$ with a very strong coefficient and with the sign that we need. Moreover, since the number $\frac{D^{\prime}}{2}\left|w_{2 n-1}^{*}(\bar{x})\right|$ is very big and the approximated element $\frac{D^{\prime}}{2} w_{2 n-1}^{*}(\bar{x}) w_{2 n}$ will appear distributed in many very little portions in in the elements $x_{n}^{*}(\bar{x}) x_{n}$, by means of subsums of $\sum_{n=1}^{\infty} x_{n}^{*}(\bar{x}) x_{n}$ we can get $w_{2 n}$ with the coefficient that we need and also with the precision that we need.

\subsection{The associated generating biorthogonal system in the complex case}

In the complex case the procedures of GBST (theorem 1 of subsection 2.1) and of MGBS (remark 2 of subsection 2.1) to the complex case cannot work, since the coefficients of the elements are not with the simple choice of signs "in a large sense" $\left(e^{i \pi}=-1, e^{i 0}=+1\right)$, but with the choice of signs $\left(e^{i \theta}: 0 \leq \theta<2 \pi\right)$; hence a more complex procedure is necessary. Analogously also for MGBS. there is a modification of the 
construction. See the observations 1 and 2 for the explanations of the modifications.

In GBST and MGBS of [2] a first index $m$ appears, in order to use the statements of the theorem directly in the many applications that followed in the work. Now we will omit this index.

We also point out that, in the proof of this theorem, we mainly shed light on the ideas; we will also go into details but only apart from the cases when they are similar to those of [2].

\section{CONSTRUCTION (C)}

In what follows $M^{\prime}$ and $Q_{0}$ are the positive integers $M^{\prime}$ and $Q$ of theorem 4 of subsection 2.1 of [2], while $N$ is another positive integer. We start from $\left(\left(u_{n}, u_{n}^{*}\right) \cup\left(e_{n, k}, e_{n, k}^{*}\right)_{k=1}^{H_{n}}\right)_{n=1}^{P^{\prime}}$ biorthogonal in $X$, with $\left\|u_{n}\right\|=1$ and $\left\|u_{n}^{*}\right\|<K$ for $1 \leq n \leq P^{\prime},\left\|e_{n, k}\right\|=1$ and $\left\|e_{n, k}^{*}\right\|<K$ for $1 \leq k \leq H_{n}$ and $1 \leq n \leq P^{\prime}$, where we have to define the integers $H_{n}$.

Then again, as in theorem 4 of subsection 2.1 of [2], we set, for each positive number $a$ and for each positive integers $q$ and $m, E X[a, 1, q]=a^{Q_{0} \cdot q}$ and $E X[a, m+1, q]=E X[a, 1, E X[a$, $m, q]]=a^{Q_{0} E X[a, m, q]}$, moreover $H_{1}=E X\left[2 K, M^{\prime}, P^{\prime}\right]=$ $(2 K)^{Q_{0} E X\left[2 K, M^{\prime}-1, P^{\prime}\right]}$ and $H_{n}=E X\left[2 K, n H_{n-1}, P\right]$ for $2 \leq n \leq P^{\prime}$, we set $P=\sum_{n=1}^{P^{\prime}}\left(H_{n}+1\right)$.

(A). We pass to define the integers $B_{n, k, 1, s}$ and the numbers $\varphi_{n, k, r, s}$. We set, for $n=1,1 \leq k \leq H_{1}+1$ and $s=1, B_{1, k, 1,1}=1$ and $\varphi_{1, k, 1,1}=$ $\frac{\pi}{20} \frac{1}{k}$, moreover, for $2 \leq s \leq k, B_{1, k, 1, s}=E X\left[2 K, 2^{N P^{2}(P-k+1)}+\right.$ $2 N(s-2), P]$ and $\varphi_{1, k, 1, s}=\frac{\pi}{20} \frac{s}{k}$; passing to $n$ with $2 \leq n \leq P^{\prime}$ we set, for $r=1,1 \leq k \leq H_{n}+1$ and $2 \leq s \leq k, B_{n, k, 1, s}=$ $E X\left[2 K, 2^{N P^{2}\left(P-\sum_{j=1}^{n-1}\left(H_{j}+1\right)-k+1\right)}+2 N(s-2), P\right]$ and $\varphi_{n, k, 1, s}=$ $\frac{\pi}{20}\left(s /\left(\sum_{j=1}^{n-1}\left(H_{j}+1\right)+k\right)\right)$; while, for $2 \leq r \leq n-1, B_{n, k, r, s}=$ $E X\left[2 K, 2^{N P^{2}\left(P-\sum_{j=1}^{n-1}\left(H_{j}+1\right)-k+1\right)}+2 M^{\prime}\left(\sum_{j=1}^{r-1}\left(H_{j}+1\right)+s-2\right), P\right]$ and $\varphi_{n, k, r, s}=\frac{\pi}{20}\left(\sum_{j=1}^{r-1}\left(H_{j}+1\right)+s\right) /\left(\sum_{j=1}^{n-1}\left(H_{j}+1\right)+k\right)$ for $1 \leq$ $s \leq H_{r}+1$, whereas for $r=n$ the only difference is that $1 \leq s \leq k$; finally $A_{n, k, r, s}=\frac{B_{n, k, r, s}}{e^{i \varphi n, k, r, s}}$ and we point out that $\frac{\pi}{20} \frac{1}{H} \leq \varphi_{n, k, r, s} \leq \frac{\pi}{20}$. 
(B). (i) We set $\left(w_{n}, w_{n}^{*}\right)_{n=1}^{P}=\left(\left(w_{n, k}, w_{n, k}^{*}\right)_{k=1}^{H_{n}+1}\right)_{n=1}^{P^{\prime}}$ biorthogonal where $w_{1,1}=\frac{u_{1}}{A_{1,1,1,1}}$ and $w_{1, k}=\frac{u_{1}}{A_{1, k, 1,1}}+\sum_{s=1}^{k-1} \frac{e_{1, s}}{A_{1, k, 1, s+1}}$ for $2 \leq k \leq H_{1}+1$; moreover, for $2 \leq n \leq P, w_{n, 1}=\sum_{r=1}^{n-1}\left(\frac{u_{r}}{A_{n, 1, r, 1}}+\right.$ $\left.\sum_{s=1}^{H_{r}} \frac{e_{r, s}}{A_{n, 1, r, s+1}}\right)+\frac{u_{n}}{A_{n, 1, n, 1}}$, while $w_{n, k}=\sum_{r=1}^{n-1}\left(\frac{u_{r}}{A_{n, k, r, 1}}+\right.$ $\left.\sum_{s=1}^{H_{r}} \frac{e_{r, s}}{A_{n, k, r, s+1}}\right)+\left(\frac{u_{n}}{A_{n, k, n, 1}}+\sum_{s=1}^{k-1} \frac{e_{n, s}}{A_{n, k, n, s+1}}\right)$ for $2 \leq k \leq H_{n}+1$; (ii) we write $(n, k)<\left(n^{\prime}, k^{\prime}\right)$ if either $n<n^{\prime}$ or $n=n^{\prime}$ but $k<k^{\prime}$.

Theorem 3 In Construction $C$ fix $\bar{x} \in X$ with $\|\bar{x}\|=1$ and let

$\bar{n}$ be an index, with $1 \leq \bar{n} \leq P-1$, so that $\left|u_{\bar{n}+1}^{*}(\bar{x})\right|>\frac{1}{Q^{2}}$.

Then for $Q$ (of (5) of theorem 4 of subsection 2.1 of [2]) sufficiently large there exists a strictly increasing sequence of indices

$$
\left.\left(((h(n, k, i), f(n, k, i)))_{i=1}^{4}\right)_{k=1}^{Q_{n}+1}\right)_{n=1}^{\bar{n}-1} \cup((h(\bar{n}, 1, i), f(\bar{n}, 1, i)))_{i=1}^{4}
$$

so that, if $\bar{u} \in \operatorname{span}\left(u_{n}\right)_{n=1}^{\bar{n}}$ with $\|\bar{u}\|=1$, bence

$$
\bar{u}=\sum_{n=1}^{\bar{n}} \bar{a}_{n} u_{n} \text { with }\left|\bar{a}_{n}\right|<K \text { for } 1 \leq n \leq \bar{n},
$$

there will exist a sequence $\left.\left.(\bar{b}(n, k, i))_{i=1}^{4}\right)_{k=1}^{Q_{n}+1}\right)_{n=1}^{\bar{n}-1} \cup(\bar{b}(\bar{n}, 1, i))_{i=1}^{4}$ of non negative numbers so that, setting $\bar{w}=\sum_{n=1}^{\bar{n}-1} \sum_{k=1}^{Q_{n}+1} \sum_{i=1}^{4} \bar{b}(n, k, i)$.. $w_{h(n, k, i), f(n, k, i)}^{*}(\bar{x}) w_{h(n, k, i), f(n, k, i)}+\sum_{i=1}^{4} \bar{b}(\bar{n}, 1, i) w_{h(\bar{n}, 1, i), f(\bar{n}, 1, i)}^{*}(\bar{x}) .$. $w_{h(\bar{n}, 1, i), f(\bar{n}, 1, i)}$, then the following properties hold:

$$
\begin{gathered}
\|\bar{w}-\bar{u}\|<\frac{1}{2^{M \cdot Q}} ; \\
\bar{n} \leq h(n, k, i) \text { for } 1 \leq n \leq \bar{n}-1,1 \leq k \leq Q_{n}+1 \\
\text { and } 1 \leq i \leq 4, \text { analogously } \bar{n} \leq h(\bar{n}, 1, i) \\
\text { for } 1 \leq i \leq 3 \text {, finally } \bar{n}+1 \leq h(\bar{n}, 1,4) ; \\
\bar{b}(n, k, i)<\frac{1}{2^{M^{\prime} \cdot Q}} \text { for } 1 \leq n \leq \bar{n}-1, \\
1 \leq k \leq Q_{n}+1 \text { and } 1 \leq i \leq 4,
\end{gathered}
$$

analogously $\bar{b}(\bar{n}, 1, i)<\frac{1}{2^{M^{\prime} \cdot Q}}$ for $1 \leq i \leq 4$. 
Proof. We start with an elementary fact which gives the first idea of the modification of the proof of theorem 4 of subsection 2.1 of [2]. Let $\left(v_{j}\right)_{j=1}^{4}$ be a sequence of four vectors in the complex plane with $v_{j}=$ $\left|v_{j}\right| e^{i \theta_{j}}$ and $0 \leq \theta_{j}<2 \pi$ for $1 \leq j \leq 4$, such that

$$
\begin{gathered}
\theta_{j+1}+\frac{\pi}{2}-\gamma_{j}<\theta_{j}<\theta_{j+1}+\pi+\alpha_{j}-\gamma_{j} \text { with } 0<\alpha_{j}<\frac{\pi}{100 Q} \\
\text { and } \frac{\pi}{10 Q}<\gamma_{j}<\frac{\pi}{10} \text {, hence with } \frac{\pi}{2}-\frac{\pi}{10}<\frac{\pi}{2}-\gamma_{j}<\theta_{j}-\theta_{j+1} \\
<\pi+\alpha_{j}-\gamma_{j}<\pi-\frac{9 \pi}{100 Q} \text {, for each } j \text { with } 1 \leq j \leq 3 \text {; then, for }
\end{gathered}
$$
each vector $v=|v| e^{i \theta}$, there is $\left(b_{j}\right)_{j=1}^{4}$ of numbers so that $v=$ $\sum_{j=1}^{4} b_{j} v_{j}$ with $0 \leq b_{j}<A$ (two numbers of $\left(b_{j}\right)_{j=1}^{4}$ are surely $=0$ ) for $1 \leq j \leq 4$, where A depends only on $|v| \cup\left(\left|v_{j}\right|\right)_{j=1}^{4}$.

Indeed suppose $\theta_{4}=0$ (it is sufficient to consider this case), hence $\frac{\pi}{2}-$ $\gamma_{3}<\theta_{3}<\pi+\alpha_{3}-\gamma_{3}$. We know that $\theta_{3}+\frac{\pi}{2}-\gamma_{2}<\theta_{2}<\theta_{3}+\pi+\alpha_{2}$ $-\gamma_{2}$ hence (see the interval of $\left.\theta_{3}\right) \pi-\left(\gamma_{2}+\gamma_{3}\right)<\theta_{2}<2 \pi+\left(\alpha_{2}+\alpha_{3}\right)$ $-\left(\gamma_{2}+\gamma_{3}\right)$. Suppose that $\pi+\frac{\pi}{20 Q}<\theta_{2}<2 \pi+\left(\alpha_{2}+\alpha_{3}\right)-\left(\gamma_{2}+\gamma_{3}\right)$, we know by above that $\frac{\pi}{2}-\frac{\pi}{10}<\frac{\pi}{2}-\gamma_{3}<\theta_{3}<\pi+\alpha_{3}-\gamma_{3}<\pi-\frac{9 \pi}{100 Q}$, hence: if $\theta_{4}=0 \leq \theta<\theta_{3}$ then $v=b_{3} v_{3}+b_{4} v_{4}$ with $b_{3} \geq 0$ and $b_{4}>0$; if $\theta_{3} \leq \theta<\theta_{2}$ then $v=b_{2} v_{2}+b_{3} v_{3}$ with $b_{2} \geq 0$ and $b_{3}>0$ (since by (4) $\left.\frac{\pi}{2}-\frac{\pi}{10}<\theta_{2}-\theta_{3}<\pi-\frac{9 \pi}{100 Q}\right)$; if $\theta_{2} \leq \theta<2 \pi\left(=\theta_{4}+2 \pi\right)$ then $v=b_{2} v_{2}+b_{4} v_{4}$ with $b_{2}>0$ and $b_{4} \geq 0$ (since $\pi+\frac{\pi}{20 Q}<\theta_{2}<$ $\left.2 \pi+\left(\alpha_{2}+\alpha_{3}\right)-\left(\gamma_{2}+\gamma_{3}\right)<2 \pi+\frac{2 \pi}{100 Q}-\frac{2 \pi}{10 Q}=2 \pi-\frac{18 \pi}{100 Q}\right)$. Suppose that $\pi-\left(\gamma_{2}+\gamma_{3}\right)<\theta_{2} \leq \pi+\frac{\pi}{20 Q}$, since $\theta_{2}+\frac{\pi}{2}-\gamma_{1}<\theta_{1}<\theta_{2}+\pi+\alpha_{1}-\gamma_{1}$ the interval of $\theta_{2}$ implies that $\pi+\frac{\pi}{2}-\frac{3 \pi}{10}<\pi-\left(\gamma_{1}+\gamma_{2}+\gamma_{3}\right)+\frac{\pi}{2}<$ $\theta_{1}<2 \pi+\frac{\pi}{20 Q}+\alpha_{1}-\gamma_{1}<2 \pi+\frac{\pi}{20 Q}+\frac{\pi}{100 Q}-\frac{\pi}{10 Q}=2 \pi-\frac{4 \pi}{100 Q}$, hence: again as above if $0 \leq \theta<\theta_{3}$ then $v=b_{3} v_{3}+b_{4} v_{4}$ with $b_{3} \geq 0$ and $b_{4}>0$; if $\theta_{3} \leq \theta<\theta_{2}$ then $v=b_{2} v_{2}+b_{3} v_{3}$ with $b_{2} \geq 0$ and $b_{3}>0$ (always since $\left.\frac{\pi}{2}-\frac{\pi}{10}<\theta_{2}-\theta_{3}<\pi-\frac{9 \pi}{100 Q}\right)$; if $\theta_{2} \leq \theta<\theta_{1}$ then $v=b_{1} v_{1}+b_{2} v_{2}$ with $b_{1} \geq 0$ and $b_{2}>0$ (always since $\frac{\pi}{2}-\frac{\pi}{10}<\theta_{1}-\theta_{2}<\pi-\frac{9 \pi}{100}$ ); 
finally if $\theta_{1} \leq \theta<2 \pi\left(=\theta_{4}+2 \pi\right)$ then $v=b_{1} v_{1}+b_{4} v_{4}$ with $b_{1}>0$ and $b_{4} \geq 0$ (by above $\pi+\frac{\pi}{2}-\frac{3 \pi}{10}<\theta_{1}<2 \pi-\frac{4 \pi}{100 Q}$ ); which completes the proof of (4).

NOTATIONS: (i) we set $w_{n, k}^{*}(\bar{x})=\left|w_{n, k}^{*}(\bar{x})\right| e^{i \theta_{n, k}}$ for $1 \leq n \leq$ $2^{P}$ and $1 \leq k \leq Q_{n}+1$; (ii) we set, for $1 \leq n \leq P, z_{n, 1}=u_{n}$ and $z_{n, 1}^{*}=$ $u_{n}^{*}, z_{n, k}=e_{n, k-1}$ and $z_{n, k}^{*}=e_{n, k-1}^{*}$ for $2 \leq k \leq Q_{n}+1$; (iii) we write, for each set of indices $\mathrm{n}, \mathrm{k}, \mathrm{i}, \mathrm{r}$ and $\mathrm{s}$ in the respective intervals and for each couple of indices $\mathrm{p}$ and $\mathrm{q}, w^{*}(n, k, i)$ for $w_{h(n, k, i), f(n, k, i)}^{*}, \theta(n, k, i)$ for $\theta_{h(n, k, i), f(n, k, i)}, \varphi(n, k, i, r, s)$ for $\varphi_{h(n, k, i), f(n, k, i), r, s}, A(n, k, i, r, s)$ for $A_{h(n, k, i), f(n, k, i), r, s}, B(n, k, i, r, s)$ for $B_{h(n, k, i), f(n, k, i), r, s}, E_{1}(p, q)$ for $2^{N Q^{2}\left(Q-\sum_{j=1}^{p-1}\left(Q_{j}+1\right)-q+1\right)}, E_{2}(p, q)$ for $2 N\left(\sum_{j=1}^{p-1}\left(Q_{j}+1\right)+q-2\right)$, finally $E_{1}(n, k, i)$ for $2^{\left.N Q^{2}\left(Q-\sum_{j=1}^{h(n, k, i)-1}\left(Q_{j}+1\right)-f(n, k, i)+1\right)\right)}=$ $E_{1}(h(n, k, i), f(n, k, i))$.

By (i) of (B) of Construction $C$ and by (ii) of Notations it follows that, for $1 \leq n \leq P$ and $1 \leq k \leq Q_{n}+1$, if $n=1 w_{1, k}=\sum_{s=1}^{k} \frac{z_{1, s}}{A_{1, k, 1, s}}$ while, for $2 \leq n \leq P, w_{n, k}=\sum_{r=1}^{n-1} \sum_{s=1}^{Q_{n}+1} \frac{z_{r, s}}{A_{n, k, r, s}}+\sum_{s=1}^{k} \frac{z_{n, s}}{A_{n, k, n, s}}$; hence proceeding as for (10.1) and (10.2) of the proof of theorem 4 of subsection 2.1 of [2] we have that $z_{P, Q_{P}+1}^{*}=\frac{w_{P, Q_{P}+1}^{*}}{A_{P, Q_{P}+1, P, Q_{P}+1}}$ and $z_{P, k}^{*}=$ $\sum_{s=k}^{Q_{P}+1} \frac{w_{P s}^{*}}{A_{P, s, P, k}}$ for $1 \leq k \leq Q_{P}$; in general we have that, for $1 \leq n \leq$ $P-1$ and $1 \leq k \leq Q_{n}+1$,

$$
\begin{gathered}
z_{n, k}^{*}=\sum_{s=k}^{Q_{n}+1} \frac{w_{n, s}^{*}}{A_{n, s, n, k}}+\sum_{r=n+1}^{P} \sum_{s=1}^{Q_{r}+1} \frac{w_{r, s}^{*}}{A_{r, s, n, k}} ; \\
\left\|w_{n, k}^{*}\right\|<\operatorname{EX}\left[2 K, E_{1}(n, k)+E_{2}(n, k)+1, Q\right]
\end{gathered}
$$

(exactly the same proof of (10.1) and (10.2) of the proof of theorem 4 of subsection 2.1 of [2] works). We affirm that there exists $((h(\bar{n}, 1, i)$, $f(\bar{n}, 1, i)))_{i=1}^{4}$ of indices so that $(\bar{n}+1,1) \leq(h(\bar{n}, 1,4), f(\bar{n}, 1,4))$, hence

$$
\bar{n}+1 \leq h(\bar{n}, 1,4) \leq P \text { and } 1 \leq f(\bar{n}, 1,4) \leq Q_{h(\bar{n}, 1,4)}+1,
$$




$$
\begin{aligned}
& \left|\frac{w_{h(\bar{n}, 1,4), f(\bar{n}, 1,4)}^{*}(\bar{x})}{A_{h(\bar{n}, 1,4), f(\bar{n}, 14), \bar{n}+1,1}}\right|=\frac{\left|w^{*}(\bar{n}, 1,4)(\bar{x})\right|}{B(\bar{n}, 1,4, \bar{n}+1,1)}>\frac{1}{Q^{3}}, \\
& \left|w^{*}(\bar{n}, 1,4)(\bar{x})\right| / B\left(\bar{n}, 1,4, \bar{n}, Q_{\bar{n}}+1\right)> \\
& Q^{2} E X\left[2 K, E_{1}(\bar{n}, 1,4)+E_{2}(\bar{n}+1,1)-1, Q\right] \text { while } \\
& \sum_{s=f(\bar{n}, 1,4)+1}^{Q_{h(\bar{n}, 1,4)}+1} \frac{\left|w_{h(\bar{n}, 1,4), s}^{*}(\bar{x})\right|}{B_{h(\bar{n}, 1,4), s, \bar{n}, Q_{\bar{n}}+1}}+\sum_{r=h(\bar{n}, 1,4)+1}^{P} \sum_{s=1}^{Q_{r}+1} \frac{\left|w_{r, s}^{*}(\bar{x})\right|}{B_{, r, s, \bar{n}, Q, \bar{n}}+1} \\
& <\operatorname{EX}\left[2 K, E_{1}(\bar{n}, 1,4)+E_{2}(\bar{n}+1,1)-2, Q\right] ; \\
& \text { moreover, for } 1 \leq i \leq 3,\left(\bar{n}, Q_{\bar{n}}+1+(i-3)\right) \leq \\
& (h(\bar{n}, 1, i), f(\bar{n}, 1, i))<(h(\bar{n}, 1, i+1), f(\bar{n}, 1, i+1)) \text { (hence } \bar{n} \leq \\
& h(\bar{n}, 1, i)) \text { with }\left|w^{*}(\bar{n}, 1, i)(\bar{x})\right| / B\left(\bar{n}, 1, i, \bar{n}, Q_{\bar{n}}+1+(i-3)\right) \\
& >\left(1 / Q^{2}\right)\left|w^{*}(\bar{n}, 1, i+1)(\bar{x})\right| / B\left(\bar{n}, 1, i+1, \bar{n}, Q_{\bar{n}}+1+(i-3)\right) \\
& >E X\left[2 K, E_{1}(\bar{n}, 1, i+1)+E_{2}\left(\bar{n}, Q_{\bar{n}}+1+(i-2)\right)-1, Q\right], \\
& \text { finally } \theta(\bar{n}, 1, i+1)+\frac{\pi}{2}-\gamma_{\bar{n}, 1, i}<\theta(\bar{n}, 1, i)<\theta(\bar{n}, 1, i+1)+\pi \\
& +\alpha_{\bar{n}, 1, i}-\gamma_{\bar{n}, 1, i} \text { where } 0<\alpha_{\bar{n}, 1, i}<\frac{\pi}{100 Q}<\frac{\pi}{20 Q}<\gamma_{\bar{n}, 1, i}<\frac{\pi}{10}, \\
& \text { in particular } \gamma_{\bar{n}, 1, i}=\varphi\left(\bar{n}, 1, i, \bar{n}, Q_{\bar{n}}+1\right)-\varphi\left(\bar{n}, 1, i+1, \bar{n}, Q_{\bar{n}}+1\right) \\
& =\varphi_{h(\bar{n}, 1, i), f(\bar{n}, 1, i), \bar{n}, Q_{\bar{n}}+1}-\varphi_{h(\bar{n}, 1, i+1), f(\bar{n}, 1, i+1), \bar{n}, Q_{\bar{n}}+1} \\
& =\left(\frac{\pi}{20} \sum_{j=1}^{\bar{n}}\left(Q_{j}+1\right)\right)\left(1 /\left(\sum_{j=1}^{h(\bar{n}, 1, i)-1}\left(Q_{j}+1\right)+f(\bar{n}, 1, i)\right)\right. \\
& \left.-1 /\left(\sum_{j=1}^{h(\bar{n}, 1, i+1)-1}\left(Q_{j}+1\right)+f(\bar{n}, 1, i+1)\right)\right) .
\end{aligned}
$$

Indeed the first part of (6.1) is true otherwise, by (1), (ii) of Notations and (5.1), $\frac{1}{Q^{2}}<\left|u_{\bar{n}+1}^{*}(\bar{x})\right|=\left|z_{\bar{n}+1,1}^{*}(\bar{x})\right|=\mid \sum_{s=1}^{Q_{\bar{n}+1}+1} \frac{w_{\bar{n}+1, s}^{*}(\bar{x})}{A_{\bar{n}+1, s, \bar{n}+1,1}}+$ $\sum_{r=\bar{n}+2}^{P} \sum_{s=1}^{Q_{r}+1} \frac{w_{r, s}^{*}(\bar{x})}{A_{r, s, \bar{n}+1,1}} \mid \leq \sum_{s=1}^{Q_{\bar{n}+1}+1} \frac{\left|w_{\bar{n}+1, s}^{*}(\bar{x})\right|}{B_{\bar{n}+1, s, \bar{n}+1,1}}+\sum_{r=\bar{n}+2}^{P} \sum_{s=1}^{Q_{r}+1}$ 
$\frac{\left|w_{r, s}^{*}(\bar{x})\right|}{B_{r, s, \bar{n}+1,1}} \leq \frac{Q_{\bar{n}+1}+1+\sum_{r=\bar{n}+2}^{P}\left(Q_{r}+1\right)}{Q_{m}^{3}} \leq \frac{1}{Q^{2}}$; about the second part of (i) we have, by (A) of Construction $C$, by (iii) of Notations and by the first part, that $\frac{\left|w^{*}(\bar{n}, 1,4)(\bar{x})\right|}{B\left(\bar{n}, 1,4, \bar{n}, Q_{\bar{n}}+1\right)}=\frac{B(\bar{n}, 1,4, \bar{n}+1,1)}{B\left(\bar{n}, 1,4, \bar{n}, Q_{\bar{n}}+1\right)} \frac{\left|w^{*}(\bar{n}, 1,4)(\bar{x})\right|}{B(\bar{n}, 1,4, \bar{n}+1,1)} \quad>$ $\frac{1}{Q^{3}} \frac{B(\bar{n}, 1,4, \bar{n}+1,1)}{B\left(\bar{n}, 1,4, \bar{n}, Q_{\bar{n}}+1\right)}=\frac{1}{Q^{3}} \frac{E X\left[2 K, E_{1}(\bar{n}, 1,4)+E_{2}(\bar{n}+1,1), Q\right]}{E X\left[2 K, E_{1}(\bar{n}, 1,4)+E_{2}\left(\bar{n}, Q_{n}+1\right), Q\right]}=$ $\frac{1}{Q^{3}} \frac{E X\left[2 K, E_{1}(\bar{n}, 1,4)+2 N\left(\sum_{j=1}^{\bar{n}}\left(Q_{j}+1\right)+1-2\right), Q\right]}{E X\left[2 K, E_{1}(\bar{n}, 1,4)+2 N\left(\sum_{j=1}^{\bar{n}}\left(Q_{j}+1\right)-2\right), Q\right]}>Q^{2} E X\left[2 K, E_{1}(\bar{n}, 1,4)+\right.$ $\left.2 N\left(\sum_{j=1}^{\bar{n}}\left(Q_{j},+1\right)+1-2\right)-1, Q\right]=Q^{2} E X\left[2 K, E_{1}(\bar{n}, 1,4)+\right.$ $\left.E_{2}(\bar{n}+1,1)-1, Q\right]$; while, about the last part of (6.1), by (5.2) we have that $\sum_{s=f(\bar{n}, 1,4)+1}^{Q_{h(\bar{n}, 1,4)}+1} \frac{\left|w_{h(\bar{n}, 1,4), s}^{*}(\bar{x})\right|}{B_{h(\bar{n}, 1,4), s, \bar{n}, Q_{\bar{n}}+1}}+\sum_{r=h(\bar{n}, 1,4)+1}^{P} \sum_{s=1}^{Q_{r}+1} \frac{\left|w_{r, s}^{*}(\bar{x})\right|}{B_{r, s, \bar{n}, Q_{\bar{n}}+1}}<$ $\sum_{s=f(\bar{n}, 1,4)+1}^{Q_{h(\bar{n}, 1,4)}+1}\left\|w_{h(\bar{n}, 1,4), s}^{*}\right\|+\sum_{r=h(\bar{n}, 1,4)+1}^{P} \sum_{s=1}^{Q_{r}+1}\left\|w_{r, s}^{*}\right\| \leq\left(Q_{h(\bar{n}, 1,4)}-\right.$ $\left.f(\bar{n}, 1,4)+1+\sum_{r=h(\bar{n}, 1,4)+1}^{P}\left(Q_{r}+1\right)\right)\left\|w_{h(\bar{n}, 1,4), f(\bar{n}, 1,4)+1}^{*}\right\|<$ $Q . . E X\left[2 K, E_{1}(h(\bar{n}, 1,4), f(\bar{n}, 1,4)+1)+E_{2}(h(\bar{n}, 1,4), f(\bar{n}, 1\right.$, $4)+1), Q]<E X\left[2 K, E_{1}(\bar{n}, 1,4)+E_{2}(\bar{n}+1,1)-2, Q\right]$ (since $\left.E_{1}(h(\bar{n}, 1,4), f(\bar{n}, 1,4)+1)<E_{1}(\bar{n}, 1,4)\right)$. On the other hand by the beginning of Construction $C$ and by (5.1) we have that $K>$ $\left|z_{\bar{n}, Q_{\bar{n}}+1}^{*}(\bar{x})\right|=\left|\frac{w_{\bar{n}, Q_{\bar{n}}+1}^{*}(\bar{x})}{A_{\bar{n}, Q_{\bar{n}}+1, \bar{n}, Q_{\bar{n}}+1}}+\sum_{r=\bar{n}+1}^{P} \sum_{s=1}^{Q_{r}+1} \frac{w_{r, s}^{*}(\bar{x})}{A_{r, s, \bar{n}, Q_{\bar{n}}+1}}\right|$, by the last two parts of (6.1) $\mid \frac{w_{\bar{n}, Q_{\bar{n}}+1}^{*}(\bar{x})}{A_{\bar{n}, Q_{m, n}+1, \bar{n}, Q_{m, \bar{n}}+1}}+\sum_{r=\bar{n}+1}^{h(\bar{n}, 1,4)-1} \sum_{s=1}^{Q_{m, r}+1}$ $\frac{w_{r, s}^{*}(\bar{x})}{A_{r, s, \bar{n}, Q_{\bar{n}}+1}}+\sum_{s=1}^{f(\bar{n}, 1,4)} \frac{w_{h(\bar{n}, 1,4), s}^{*}(\bar{x})}{A_{h(\bar{n}, 1,4), s, \bar{n}, Q_{\bar{n}}+1}}|<K+| \sum_{s=f(\bar{n}, 1,4)+1}^{Q_{h(\bar{n}, 1,4}+1}$ $\frac{w_{h(\bar{n}, 1,4), s}^{*}(\bar{x})}{A_{h(\bar{n}, 1,4), s, \bar{n}, Q_{\bar{n}}+1}}+\sum_{r=h(\bar{n}, 1,4)+1}^{P} \sum_{s=1}^{Q_{r}+1} \frac{w_{r, s}^{*}(\bar{x})}{A_{r, s, \bar{n}, Q_{\bar{n}}+1}} \mid<2 E X[2 K$, $\left.E_{1}(\bar{n}, 1,4)+E_{2}(\bar{n}+1,1)-2, Q\right]=\frac{2}{Q^{2}} \cdot \frac{E X\left[2 K, E_{1}(\bar{n}, 1,4)+E_{2}(\bar{n}+1,1)-2, Q\right]}{E X\left[2 K, E_{1}(\bar{n}, 1,4)+E_{2}(\bar{n}+1,1)-1, Q\right]}$ $Q^{2} \operatorname{EX}\left[2 K, E_{1}(\bar{n}, 1,4)+E_{2}(\bar{n}+1,1)-1, Q\right]<\frac{2}{Q^{2}}$ $\frac{E X\left[2 K, E_{1}(\bar{n}, 1,4)+E_{2}(\bar{n}+1,1)-2, Q\right]}{E X\left[2 K, E_{1}(\bar{n}, 1,4)+E_{2}(\bar{n}+1,1)-1, Q\right]} \frac{\left|w^{*}(\bar{n}, 1,4)(\bar{x})\right|}{B\left(\bar{n}, 1,4, \bar{n}, Q_{\bar{n}}+1\right)}<\frac{1}{2^{Q}} \frac{\left|w^{*}(\bar{n}, 1,4)(\bar{x})\right|}{B\left(\bar{n}, 1,4, \bar{n}, Q_{\bar{n}}+1\right)}$.

It follows that there exists $(h(\bar{n}, 1,3), f(\bar{n}, 1,3))$ with $\left(\bar{n}, Q_{\bar{n}}+1\right)$ $\leq(h(\bar{n}, 1,3), f(\bar{n}, 1,3))<(h(\bar{n}, 1,4), f(\bar{n}, 1,4))$ (hence $\bar{n} \leq$ 
$h(\bar{n}, 1,3))$ so that

$$
\begin{aligned}
& \frac{\left|w^{*}(\bar{n}, 1,3)(\bar{x})\right|}{B\left(\bar{n}, 1,3, \bar{n}, Q_{\bar{n}}+1\right)}>\frac{1}{Q^{2}} \frac{\left|w^{*}(\bar{n}, 1,4)(\bar{x})\right|}{B\left(\bar{n}, 1,4, \bar{n}, Q_{\bar{n}}+1\right)} \\
& >\text { (by (6.1)) } E X\left[2 K, E_{1}(\bar{n}, 1,4)\right. \\
& \left.+E_{2}(\bar{n}+1,1)-1, Q\right] \text {; } \\
& \vartheta(\bar{n}, 1,4)+\varphi\left(\bar{n}, 1,4, \bar{n}, Q_{\bar{n}}+1\right)+\frac{\pi}{2}<\vartheta(\bar{n}, 1,3) \\
& +\varphi\left(\bar{n}, 1,3, \bar{n}, Q_{\bar{n}}+1\right)<\vartheta(\bar{n}, 1,4)+\varphi\left(\bar{n}, 1,4, \bar{n}, Q_{\bar{n}}+1\right) \\
& +\pi+\alpha_{\bar{n}, 1,3} \text { where } 0<\alpha_{\bar{n}, 1,3}<\frac{\pi}{100 Q}
\end{aligned}
$$

(let us deepen (7.2), on $\alpha_{\bar{n}, 1,3}$ : setting $P^{\prime}=1+\sum_{r=\bar{n}+1}^{h(\bar{n}, 1,4)-1}\left(Q_{r}+1\right)+$ $f(\bar{n}, 1,4)$ and $\left(v_{n}\right)_{n=1}^{P^{\prime}}=\frac{w_{\bar{n}, Q_{\bar{n}}+1}^{*}(\bar{x})}{A_{\bar{n}, Q_{\bar{n}}+1, \bar{n}, Q_{\bar{n}}+1}} \cup\left(\left(\frac{w_{r, s}^{*}(\bar{x})}{A_{r, s, \bar{n}, Q_{\bar{n}}+1}}\right)_{s=1}^{Q_{r}+1}\right)_{r=\bar{n}+1}^{h(\bar{n}, 1,4)-1} \cup$ $\left(\frac{w_{h(\bar{n}, 1,4), s}^{*}(\bar{x})}{A_{h(\bar{n}, 1,4), s, \bar{n}, Q_{\bar{n}}+1}}\right)_{s=1}^{f(\bar{n}, 1,4)}$, let $Q^{\prime}$ be an integer with $Q^{\prime}>P^{\prime}$ and suppose that $\left|\sum_{n=1}^{P^{\prime}} v_{n}\right|<\left|v_{P^{\prime}}\right| / 2^{Q^{\prime}}$, suppose moreover that there exists $n^{\prime}$ with $1 \leq n^{\prime} \leq P^{\prime}-1$ with $v_{n^{\prime}}=\left|v_{n^{\prime}}\right| e^{i \vartheta_{n^{\prime}}},\left|v_{n^{\prime}}\right|>\left|v_{P^{\prime}}\right| / 2 P^{\prime}$ and $\vartheta_{n^{\prime}}=\pi+\alpha$ with $0<\alpha<\pi$ where $\vartheta_{P^{\prime}}=0$, suppose also that for each $\mathrm{n}$ with $1 \leq n \leq P^{\prime}-1$ such that $\left|v_{n}\right|>\left|v_{P^{\prime}}\right| / 2 P^{\prime}$ then $v_{n}=\left|v_{n}\right| e^{i \vartheta_{n}}$ with $\pi+\alpha \leq \vartheta_{n} \leq 2 \pi$, then the fact that $\left|\sum_{n=1}^{P^{\prime}} v_{n}\right|<\left|v_{P^{\prime}}\right| / 2^{Q^{\prime}}$ implies the fact that $\left|v_{n^{\prime}}\right| \operatorname{sen} \alpha<\left|v_{P^{\prime}}\right| / 2^{Q^{\prime}}$ that is $\operatorname{sen} \alpha<\left(1 /\left|v_{n^{\prime}}\right|\right)\left(\left|v_{P^{\prime}}\right| / 2^{Q^{\prime}}\right)<$ $\left(2 P^{\prime}\right) / 2^{Q \prime}$, we conclude that for sufficiently large $Q$ we can suppose in our case $0<\alpha_{\bar{n}, 1,3}<\pi /(100 Q)$ ).

At this point we repeat the whole procedure starting from $(h(\bar{n}$, $1,3), f(\bar{n}, 1,3))$ and $\left(\bar{n}, Q_{\bar{n}}+1\right)$ instead of $(h(\bar{n}, 1,4), f(\bar{n}, 1,4))$ and $(\bar{n}+1,1)$, then we get $(h(\bar{n}, 1,2), f(\bar{n}, 1,2))$ with $\left(\bar{n}, Q_{\bar{n}}\right) \leq(h(\bar{n}$, $1,2), f(\bar{n}, 1,2))<(h(\bar{n}, 1,3), f(\bar{n}, 1,3))$ (hence again $\bar{n} \leq h(\bar{n}, 1,2))$ such that $\frac{\left|w^{*}(\bar{n}, 1,2)(\bar{x})\right|}{B\left(\bar{n}, 1,2, \bar{n}, Q_{\bar{n}}\right)}>\frac{1}{Q^{2}} \frac{\left|w^{*}(\bar{n}, 1,3)(\bar{x})\right|}{B\left(\bar{n}, 1,3, \bar{n}, Q_{\bar{n}}\right)}>\operatorname{EX}\left[2 K, E_{1}(\bar{n}, 1,3)+\right.$ $\left.E_{2}\left(\bar{n}, Q_{\bar{n}}+1\right)-1, Q\right]$ and with $\vartheta(\bar{n}, 1,3)+\varphi\left(\bar{n}, 1,3, \bar{n}, Q_{m, \bar{n}}\right)+\frac{\pi}{2}<$ $\vartheta(\bar{n}, 1,2)+\varphi\left(\bar{n}, 1,2, \bar{n}, Q_{m, \bar{n}}\right)<<\vartheta(\bar{n}, 1,3)+\varphi\left(\bar{n}, 1,3, \bar{n}, Q_{\bar{n}}\right)+\pi+$ $\alpha_{\bar{n}, 1,2}$ and again $0<\alpha_{\bar{n}, 1,2}<\frac{\pi}{100 Q}$ (indeed by (6.1) and by (7.1) we have 
that $\frac{1}{Q^{2}} \frac{\left|w^{*}(\bar{n}, 1,3)(\bar{x})\right|}{B\left(\bar{n}, 1,3, \bar{n}, Q_{\bar{n}}\right)}=\frac{1}{Q^{2}} \frac{B\left(\bar{n}, 1,3, \bar{n}, Q_{\bar{n}}+1\right)}{B\left(\bar{n}, 1,3, \bar{n}, Q_{\bar{n}}\right)} \frac{\left|w^{*}(\bar{n}, 1,3)(\bar{x})\right|}{B\left(\bar{n}, 1,3, \bar{n}, Q_{\bar{n}}+1\right)} \quad>$ $\frac{B\left(\bar{n}, 1,3, \bar{n}, Q_{\bar{n}}+1\right)}{B\left(\bar{n}, 1,3, \bar{n}, Q_{\bar{n}}\right)} . . E X\left[2 K, E_{1}(\bar{n}, 1,4)+E_{2}(\bar{n}+1,1)-1, Q\right]=$ $\frac{E X\left[2 K, E_{1}(\bar{n}, 1,3)+E_{2}\left(\bar{n}, Q_{\bar{n}}+1\right), Q\right]}{E X\left[2 K, E_{1}(\bar{n}, 1,3)+E_{2}\left(\bar{n}, Q_{\bar{n}}\right), Q\right]} \cdot E X\left[2 K, E_{1}(\bar{n}, 1,4)+E_{2}(\bar{n}+1,1)-\right.$ $\left.1, Q]>\operatorname{EX}\left[2 K, E_{1}(\bar{n}, 1,3)+E_{2}\left(\bar{n}, Q_{\bar{n}}+1\right)-1, Q\right]\right)$. Since $(h(\bar{n}$, $1,3), f(\bar{n}, 1,3))<(h(\bar{n}, 1,4), f(\bar{n}, 1,4))$ by the last part of (A) of Construction $C$ and by (iii) of Notations we have that $\varphi\left(\bar{n}, 1,3, \bar{n}, Q_{\bar{n}}+1\right)=$ $\varphi\left(\bar{n}, 1,4, \bar{n}, Q_{\bar{n}}+1\right)+\gamma_{\bar{n}, 1,3}$ with $\gamma_{\bar{n}, 1,3}=\frac{\pi}{20} \sum_{j=1}^{\bar{n}}\left(Q_{, j}+1\right)(1 /$ $\left(\sum_{j=1}^{h(\bar{n}, 1,3)-1}\left(Q_{j}+1\right)+f(\bar{n}, 1,3)\right)-1 /\left(\sum_{j=1}^{h(\bar{n}, 1,4)-1}\left(Q_{j}+1\right)+f(\bar{n}\right.$, $1,4)))>\frac{\pi}{10 Q}$, hence, by $(7.2), \vartheta(\bar{n}, 1,4)+\frac{\pi}{2}-\gamma_{\bar{n}, 1,3}<\vartheta(\bar{n}, 1,3)<$ $\vartheta(\bar{n}, 1,4)+\pi+\alpha_{\bar{n}, 1,3}-\gamma_{\bar{n}, 1,3}$; analogously $\varphi\left(\bar{n}, 1,2, \bar{n}, Q_{\bar{n}}\right)=\varphi(\bar{n}, 1,3$, $\left.\bar{n}, Q_{\bar{n}}\right)+\gamma_{\bar{n}, 1,2}$ with $\gamma_{\bar{n}, 1,2}>\frac{\pi}{10 Q}$, hence $\vartheta(\bar{n}, 1,3)+\frac{\pi}{2}-\gamma_{\bar{n}, 1,2}<$ $\vartheta(\bar{n}, 1,2)<\vartheta(\bar{n}, 1,3)+\pi+\alpha_{\bar{n}, 1,2}-\gamma_{\bar{n}, 1,2}$. Finally we repeat the whole procedure starting from $(h(\bar{n}, 1,2), f(\bar{n}, 1,2))$ and $\left(\bar{n}, Q_{\bar{n}}\right)$ instead of $(h(\bar{n}, 1,3), f(\bar{n}, 1,3))$ and $\left(\bar{n}, Q_{\bar{n}}+1\right)$, then we get $(h(\bar{n}, 1,1)$, $f(\bar{n}, 1,1))$ with $\left(\bar{n}, Q_{\bar{n}}-1\right) \leq(h(\bar{n}, 1,1), f(\bar{n}, 1,1))<(h(\bar{n}, 1$, 2), $f(\bar{n}, 1,2))$ (hence again $\bar{n} \leq h(\bar{n}, 1,1))$ such that $\frac{\left|w^{*}(\bar{n}, 1,1)(\bar{x})\right|}{B\left(\bar{n}, 1,1, \bar{n}, Q_{\bar{n}}-1\right)}>$ $\frac{1}{Q^{2}} \frac{\left|w^{*}(\bar{n}, 1,2)(\bar{x})\right|}{B\left(\bar{n}, 1,2, \bar{n}, Q_{\bar{n}}-1\right)}>E X\left[2 K, E_{1}(\bar{n}, 1,2)+E_{2}\left(\bar{n}, Q_{\bar{n}}\right)-1, Q\right]$ and with $\vartheta(\bar{n}, 1,2)+\frac{\pi}{2}-\gamma_{\bar{n}, 1,1}<\vartheta(\bar{n}, 1,1)<\vartheta(\bar{n}, 1,2)+\pi+\alpha_{\bar{n}, 1,1}-\gamma_{\bar{n}, 1,1} ;$ then (6.1) and (6.2) have been been proved.

So proceeding we have that there exists also a strictly increasing sequence $\left.\left(((h(n, k, i), f(n, k, i)))_{i=1}^{4}\right)_{k=1}^{Q_{n}+1}\right)_{n=1}^{\bar{n}-1}$ with the properties of (3.2), moreover, for each $\mathrm{n}$ with $1 \leq n \leq \bar{n}-1$ with the following PROPERTIES:

Let us set , for $1 \leq k \leq Q_{n}+1$ and $1 \leq i \leq 4$, moreover for $1 \leq$ $n \leq \bar{n}-2, N(n, k, i)=\sum_{j=n+1}^{\bar{n}=1} 4\left(Q_{j}+1\right)+4\left(\bar{Q}_{n}-k+1\right)+(5-i)$, while $N(\bar{n}-1, k, i)=4\left(Q_{\bar{n}-1}-k+1\right)+(5-i)$, for $1 \leq k \leq Q_{\bar{n}-1}+1$ and $1 \leq i \leq 4$ (hence $N\left(\bar{n}-1, Q_{\bar{n}-1}+1, i\right)=5-i$ for $1 \leq i \leq 3$ and $\left.N\left(\bar{n}-1, Q_{\bar{n}-1}+1,4\right)=1\right)$;

Then we have that:(i) for $1 \leq k \leq Q_{n}+1$ and $1 \leq i \leq 3,\left(\bar{n}, Q_{\bar{n}}-\right.$ $1-N(n, k, i)) \leq(h(n, k, i), f(n, k, i))<(h(n, k, i+1), f(n, k, i+1))$, $\left|w^{*}(n, k, i)(\bar{x})\right| / B\left(n, k, i, \bar{n}, Q_{\bar{n}}-1-N(n, k, i)\right)>E X\left[2 K, E_{1}(n\right.$, $\left.k, i+1)+E_{2}\left(\bar{n}, Q_{\bar{n}}-N(n, k, i)\right)-1, Q\right], \theta(n, k, i+1)+\frac{\pi}{2}-\gamma_{n, k, i}<$ $\vartheta(n, k, i)<\vartheta(n, k, i+1)+\pi+\alpha_{n, k, i}-\gamma_{n, k, i}$; (ii) for $1 \leq k \leq Q_{n}$, 
$\left(\bar{n}, Q_{\bar{n}}-1-N(n, k, 4)\right) \leq(h(n, k, 4), f(n, k, 4))<(h(n, k+1,1)$, $f(n, k+1,1))$ and $\left|w^{*}(n, k, 4)(\bar{x})\right| / B\left(n, k, 4, \bar{n}, Q_{\bar{n}}-1-N(n, k, 4)\right)>$ $E X\left[2 K, E_{1}(n, k+1,1)+E_{2}\left(\bar{n}, Q_{\bar{n}}-N(n, k, 4)\right)-1, Q\right]$;

(iii) for $1 \leq n \leq \bar{n}-2,\left(\bar{n}, Q_{\bar{n}}-1-N\left(n, Q_{n}+1,4\right)\right) \leq(h(n$, $\left.\left.Q_{n}+1,4\right), f\left(n, Q_{n}+1,4\right)\right)<(h(n+1,1,1), f(n+1,1,1))$ and $\left|w^{*}\left(n, Q_{n}+1,4\right)(\bar{x})\right| / B\left(n, Q_{n}+1,4, \bar{n}, Q_{\bar{n}}-1-N\left(n, Q_{n}+1,4\right)\right)$ $>E X\left[2 K, E_{1}(n+1,1,1)+E_{2}\left(\bar{n}, Q_{\bar{n}}-N\left(n, Q_{n}+1,4\right)\right)-1, Q\right]$;

(iv) $\left(\bar{n}, Q_{\bar{n}}-1-N\left(\bar{n}-1, Q_{\bar{n}-1}+1,4\right)\right)=\left(\bar{n}, Q_{\bar{n}}-2\right) \leq(h(\bar{n}-$ $\left.\left.1, Q_{\bar{n}-1}+1,4\right), f\left(\bar{n}-1, Q_{\bar{n}-1}+1,4\right)\right)<(h(\bar{n}, 1,1), f(\bar{n}, 1,1))$ with $\left|w^{*}\left(\bar{n}-1, Q_{\bar{n}-1}+1,4\right)(\bar{x})\right| / B\left(\bar{n}-1, Q_{\bar{n}-1}+1,4, \bar{n}, Q_{\bar{n}}-2\right)>E X[2 K$, $\left.E_{1}(\bar{n}, 1,1)+E_{2}\left(\bar{n}, Q_{\bar{n}}-1\right)-1, Q\right]$;

(v) $\bar{n} \leq h(n, k, i)$ for $1 \leq n \leq \bar{n}-1,1 \leq k \leq Q_{n}+1$ and $1 \leq i \leq 4$.

Indeed by means of the same procedure there exists $\left(h\left(\bar{n}-1, Q_{\bar{n}-1}+\right.\right.$ $\left.1,4)), f\left(\bar{n}-1, Q_{\bar{n}-1}+1,4\right)\right)$ with $\left(\bar{n}, Q_{\bar{n}}-2\right) \leq\left(h\left(\bar{n}-1, Q_{\bar{n}-1}+\right.\right.$ $\left.1,4), f\left(\bar{n}-1, Q_{\bar{n}-1}+1,4\right)\right)<(h(\bar{n}, 1,1), f(\bar{n}, 1,1))$ (hence always $\bar{n} \leq$ $\left.h\left(\bar{n}-1, Q_{\bar{n}-1}+1,4\right)\right)$ with $\frac{\left|w^{*}\left(\bar{n}-1, Q_{\bar{n}-1}+1,4\right)(\bar{x})\right|}{B\left(\bar{n}-1, Q_{\bar{n}-1}+1,4, \bar{n}, Q_{\bar{n}}-2\right)}>\frac{1}{Q^{2}} \frac{\left|w^{*}(\bar{n}, 1,1)(\bar{x})\right|}{B\left(\bar{n}, 1,1, \bar{n}, Q_{\bar{n}}-2\right)}>$ $E X\left[2 K, E_{1}(\bar{n}, 1,1)+E_{2}\left(\bar{n}, Q_{\bar{n}}-1\right)-1, Q\right]$ and with $\vartheta(\bar{n}, 1,1)+\frac{\pi}{2}-$ $\gamma_{\bar{n}-1, Q_{\bar{n}-1}+1,4}<\vartheta\left(\bar{n}-1, Q_{\bar{n}-1}+1,4\right)<\vartheta(\bar{n}, 1,1)+\pi+\alpha_{\bar{n}-1, Q_{\bar{n}-1}+1,4}-$ $\gamma_{\bar{n}-1, Q_{\bar{n}-1}+1,4}$; so proceeding there exists also $\left(\left(h\left(\bar{n}-1, Q_{\bar{n}-1}+1, i\right)\right.\right.$, $\left.\left.f\left(\bar{n}-1, Q_{\bar{n}-1}+1, i\right)\right)\right)_{i=1}^{3}$ so that, for $1 \leq i \leq 3,\left(\bar{n}, Q_{\bar{n}}-1-N(\bar{n}-\right.$ $\left.\left.1, Q_{\bar{n}-1}+1, i\right)\right)=\left(\bar{n}, Q_{\bar{n}}-1-(5-i)\right) \leq\left(h\left(\bar{n}-1, Q_{\bar{n}-1}+\right.\right.$ $\left.t 1, i), f\left(\bar{n}-1, Q_{\bar{n}-1}+1, i\right)\right)$ (hence always $\bar{n} \leq h\left(\bar{n}-1, Q_{\bar{n}-1}+\right.$ $1, i))<\left(h\left(\bar{n}-1, Q_{\bar{n}-1}+1, i+1\right), f\left(\bar{n}-1, Q_{\bar{n}-1}+1, i+1\right)\right)$ and

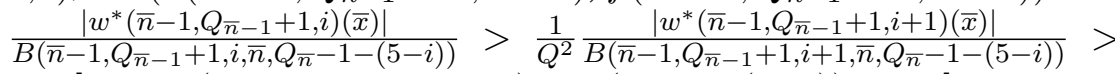
$E X\left[2 K, E_{1}\left(\bar{n}-1, Q_{\bar{n}-1}+1, i+1\right)+E_{2}\left(\bar{n}, Q_{\bar{n}}-(5-i)\right)-1, Q\right]$, moreover $\vartheta\left(\bar{n}-1, Q_{\bar{n}-1}+1, i+1\right)+\frac{\pi}{2}-\gamma_{\bar{n}-1, Q_{\bar{n}-1}+1, i}<\vartheta\left(\bar{n}-1, Q_{\bar{n}-1}+1, i\right)<$ $\vartheta\left(\bar{n}-1, Q_{\bar{n}-1}+1, i+1\right)+\pi+\alpha_{\bar{n}-1, Q_{\bar{n}-1}+1, i}-\gamma_{\bar{n}-1, Q_{\bar{n}-1}+1, i}$. Analogously we get $\left.((h(\bar{n}-1, k, i), f(\bar{n}-1, k, i)))_{i=1}^{4}\right)_{k=1}^{Q_{\bar{n}-1}}$ with, for each $\mathrm{k}$ with $1 \leq k \leq Q_{\bar{n}-1},\left(\bar{n}, Q_{\bar{n}}-1-N(\bar{n}-1, k, 4)\right)=\left(\bar{n}, Q_{\bar{n}}-1-\right.$ $\left.4\left(Q_{\bar{n}-1}-k+1\right)-1\right) \leq(h(\bar{n}-1, k, 4), f(\bar{n}-1, k, 4))<(h(\bar{n}-$ $1, k+1,1), f(\bar{n}-1, k+1,1))$ and $\frac{\left|w^{*}(\bar{n}-1, k, 4)(\bar{x})\right|}{B\left(\bar{n}-1, k, 4, \bar{n}, Q_{\bar{n}}-1-4\left(Q_{\bar{n}}-1-k+1\right)-1\right)}>$ $E X\left[2 K, E_{1}(\bar{n}-1, k+1,1)+E_{2}\left(\bar{n}, Q_{\bar{n}}-4\left(Q_{\bar{n}-1}-k+1\right)-1\right)-1, Q\right]$ while, for each i with $1 \leq i \leq 3,\left(\bar{n}, Q_{\bar{n}}-1-N(\bar{n}-1, k, i)\right)=$ 
$\left(\bar{n}, Q_{\bar{n}}-1-4\left(Q_{\bar{n}-1}-k+1\right)-(5-i)\right) \leq(h(\bar{n}-1, k, i), f(\bar{n}-$ $1, k, i))<(h(\bar{n}-1, k, i+1), f(\bar{n}-1, k, i+1))$ (hence always $\bar{n} \leq h(\bar{n}-$ $1, k, i+1)$ for $1 \leq i \leq 4)$ and $\frac{\left|w^{*}(\bar{n}-1, k, i)(\bar{x})\right|}{B\left(\bar{n}-1, k, i, \bar{n}, Q_{\bar{n}}-1-4\left(Q_{\bar{n}}-1-k+1\right)-(5-i)\right)}>$ $E X\left[2 K, E_{1}(\bar{n}-1, k, i+1)+E_{2}\left(\bar{n}, Q_{\bar{n}}-4\left(Q_{\bar{n}-1}-k\right)-9+i\right)-1, Q\right]$ (since $\left.-4\left(Q_{\bar{n}-1}-k+1\right)-(5-i)=-4\left(Q_{\bar{n}-1}-k\right)-9+i\right)$; now the procedure to complete the proof of Properties is clear.

At this point we can write in (2), by means of (ii) of Notations,

$$
\begin{gathered}
\bar{u}=\sum_{n=1}^{\bar{n}-1}\left(\bar{a}_{n, 1} u_{n}+\sum_{k=1}^{Q_{n}} \bar{a}_{n, k+1} e_{n, k}\right)+\bar{a}_{\bar{n}, 1} u_{\bar{n}}=\sum_{n=1}^{\bar{n}-1} \sum_{k=1}^{Q_{n}+1} \bar{a}_{n, k} z_{n, k} \\
+\bar{a}_{\bar{n}, 1} u_{\bar{n}} \text { with } \bar{a}_{n, k+1}=0 \text { for } 1 \leq k \leq Q_{n} \text { and } 1 \leq n \leq \bar{n}-1, \\
\text { while } \bar{a}_{n, 1}=\bar{a}_{n} \text { for } 1 \leq n \leq \bar{n} .
\end{gathered}
$$

Like theorem 2 our aim now is to approximate the addenda of (8) and, since the numbers $\bar{a}_{n, k}$ are in the complex plane, this approximation bases itself on the procedure of (4). Now the reason of the presence of the angles $\varphi_{n, k, r, s}$ in (A) of Construction $C$ appears: since without these angles (that is if $\varphi_{n, k, r, s}=0$ ) we could have, for instance in (7.2), $\theta(\bar{n}, 1,3)=\theta(\bar{n}, 1,4)+\pi$ (which is exactly (14.1) of theorem 4 of subsection 2.1 of [2], since the condition becomes $\theta(\bar{n}, 1,4)+\frac{\pi}{2}<\vartheta(\bar{n}, 1,3)<$ $\left.\vartheta(\bar{n}, 1,4)+\pi+\alpha_{\bar{n}, 1,3}\right)$, but in this case the procedure of (4) could not work. Therefore, by means of the same procedure of the proof of (17.1) of theorem 4 of subsection 2.1 of [2], we affirm that we can determine numbers as in the thesis of proposition, such that (3.2) is satisfied, moreoverso that the following analogous relation of (17.3) of theorem 2 holds:

$$
\left\|\sum_{i=1}^{4} \bar{b}(\bar{n}, 1, i) w_{h(\bar{n}, 1, i), f(\bar{n}, 1, i)}^{*}(\bar{x}) \bar{w}_{h(\bar{n}, 1, i), f(\bar{n}, 1, i)}-\bar{a}_{\bar{n}, 1} u_{\bar{n}}\right\|<\frac{1}{2^{\left(M^{\prime}+1\right) Q}} .
$$

Indeed, analogously to (17.1), (17.2) and (17.3) of theorem 4 of subsection 2.1 of [2], this inequalitybases itself on the equality

$$
\sum_{i=1}^{4} \bar{b}(\bar{n}, 1, i) w_{h(\bar{n}, 1, i), f(\bar{n}, 1, i)}^{*}(\bar{x}) \frac{1}{A_{h(\bar{n}, 1, i), f(\bar{n}, 1, i), \bar{n}, 1}}=\bar{a}_{\bar{n}, 1}
$$


that is, by (A) of Construction $C$ and by (i) of Notations,

$$
\begin{array}{r}
\sum_{i=1}^{4} \bar{b}(\bar{n}, 1, i)\left|w_{h(\bar{n}, 1, i), f(\bar{n}, 1, i)}^{*}(\bar{x})\right| \frac{e^{i\left(\theta_{h(\bar{n}, 1, i), f(\bar{n}, 1, i)}+\varphi_{h(\bar{n}, 1, i), f(\bar{n}, 1, i), \bar{n}, 1}\right)}}{B_{h(\bar{n}, 1, i), f(\bar{n}, 1, i), \bar{n}, 1}} \\
=\bar{a}_{\bar{n}, 1 .}
\end{array}
$$

At this point we notice that, in order to use the procedure of (4) in (9), we need that $(\vartheta(\bar{n}, 1, i)+\varphi(\bar{n}, 1, i, \bar{n}, 1))_{i=1}^{4}$ verify the hypothesis of $(4)$, while by $(6.2)$ we only know that $(\vartheta(\bar{n}, 1, i))_{i=1}^{4}$ verify the hypothesis of (4), precisely we know that (see the last part of (6.2)), for each i with $1 \leq i \leq 3, \theta(\bar{n}, 1, i+1)+\frac{\pi}{2}-\gamma_{\bar{n}, 1, i}<\theta(\bar{n}, 1, i)<\theta(\bar{n}, 1, i+1)+\pi+$ $\alpha_{\bar{n}, 1, i}-\gamma_{\bar{n}, 1, i}$ where $0<\alpha_{\bar{n}, 1, i}<\frac{\pi}{100 Q}$ and $\gamma_{\bar{n}, 1, i}=\alpha \sum_{j=1}^{\bar{n}}\left(Q_{j}+1\right)$ with $\alpha=\frac{\pi}{20}\left(\frac{1}{\sum_{j=1}^{h(\bar{n}, 1, i)-1}\left(Q_{j}+1\right)+f(\bar{n}, 1, i)}-\frac{1}{\sum_{j=1}^{h(\bar{n}, 1, i+1)-1}\left(Q_{j}+1+f(\bar{n}, 1, i+1)\right.}\right)$; therefore wehave to check that also $(\vartheta(\bar{n}, 1, i)+\varphi(\bar{n}, 1, i, \bar{n}, 1))_{i=1}^{4}$ verify the hypothesis of (4). Indeed we have that, setting by above $\beta_{\bar{n}, 1, i}=$ $\varphi(\bar{n}, 1, i, \bar{n}, 1)-\varphi(\bar{n}, 1, i+1, \bar{n}, 1)=\alpha\left(\sum_{j=1}^{\bar{n}-1}\left(Q_{j}+1\right)+1\right)$, then $\vartheta(\bar{n}, 1, i+1)+\varphi(\bar{n}, 1, i+1, \bar{n}, 1)+\frac{\pi}{2}-\gamma_{\bar{n}, 1, i}<\vartheta(\bar{n}, 1, i+1)+\varphi(\bar{n}, 1, i+$ $1, \bar{n}, 1)+\frac{\pi}{2}-\gamma_{\bar{n}, 1, i}+\beta_{\bar{n}, 1, i}=\theta(\bar{n}, 1, i+1)+\varphi(\bar{n}, 1, i, \bar{n}, 1)+\frac{\pi}{2}-\gamma_{\bar{n}, 1, i}<$ $\theta(\bar{n}, 1, i)+\varphi(\bar{n}, 1, i, \bar{n}, 1)<\theta(\bar{n}, 1, i+1)+\varphi(\bar{n}, 1, i, \bar{n}, 1)+\pi+\alpha_{\bar{n}, 1, i}-$ $\gamma_{\bar{n}, 1, i}=\theta(\bar{n}, 1, i+1)+\varphi(\bar{n}, 1, i+1, \bar{n}, 1)+\pi+\alpha_{\bar{n}, 1, i}-\gamma_{\bar{n}, 1, i}+\beta_{\bar{n}, 1, i}<$ $\theta(\bar{n}, 1, i+1)+\varphi(\bar{n}, 1, i+1, \bar{n}, 1)+\pi+\alpha_{\bar{n}, 1, i}-\frac{1}{2} \gamma_{\bar{n}, 1, i}$ (since $\beta_{\bar{n}, 1, i}=$ $\alpha\left(\sum_{j=1}^{\bar{n}-1}\left(Q_{j}+1\right)+1\right)<\frac{1}{2} \alpha \sum_{j=1}^{\bar{n}}\left(Q_{j}+1\right)=\frac{1}{2} \gamma_{\bar{n}, 1, i}$ by the definitions of Construction $C)$, that is $\theta(\bar{n}, 1, i+1)+\varphi(\bar{n}, 1, i+1, \bar{n}, 1)+\frac{\pi}{2}-\gamma_{\bar{n}, 1, i}<$ $\theta(\bar{n}, 1, i)+\varphi(\bar{n}, 1, i, \bar{n}, 1)<\theta(\bar{n}, 1, i+1)+\varphi(\bar{n}, 1, i+1, \bar{n}, 1)+\pi+$ $\alpha_{\bar{n}, 1, i}-\frac{1}{2} \gamma_{\bar{n}, 1, i}$; then it is possible to check that the procedure of the same proof of (4) continues to work (that is the reason is that, by the definitions of Construction $C, \sum_{j=1}^{\bar{n}-1}\left(Q_{j}+1\right)+1$ is negligible if compared with $\left.\sum_{j=1}^{\bar{n}}\left(Q_{j}+1\right)\right)$. Therefore it follows that the equality of (9) is possible.

However we have also to notice that, if $Q$ is very large, these angles $\gamma_{\bar{n}, 1, i}$ could be very little (since we know by the last part of (A) of Construction $C$ that $\frac{\pi}{20 Q}<\gamma_{\bar{n}, 1, i}<\frac{\pi}{10}$ ), which is crucial if in the second part of (7.2) we would have $\theta_{h(\bar{n}, 1,3), f(\bar{n}, 1,3)}+\varphi_{h(\bar{n}, 1,3), f(\bar{n}, 1,3), \bar{n}, Q_{\bar{n}+1}}=$ 
$\theta_{h(\bar{n}, 1,4), f(\bar{n}, 1,4)}+\varphi_{h(\bar{n}, 1,4), f(\bar{n}, 1,4), \bar{n}, Q_{\bar{n}}+1}+\pi$, hence $\theta_{h(\bar{n}, 1,3), f(\bar{n}, 1,3)}=$ $\theta_{h(\bar{n}, 1,4), f(\bar{n}, 1,4)}+\gamma_{\bar{n}, 1,3}+\pi$, indeed this fact would imply for the $\bar{b}(\bar{n}, 1, j) \frac{\left|w_{h(\bar{n}, 1, j), f(\bar{n}, 1, j)}^{*}(\bar{x})\right|}{B_{h(\bar{n}, 1, j), f(\bar{n}, 1, j), \bar{n}, 1}}$ of (9) to be very large, which seems to be at odds with (3.3) where we need for the numbers $\bar{b}(\bar{n}, 1, j)$ to be very little. But this fact is not a difficulty because, as we can see in Properties, it is only sufficient to ask to the integer $N$ (which appears in (A) of Construction $C$ ) to be sufficiently large; indeed if $N$ increases also $\left|w_{h(\bar{n}, 1, j), f(\bar{n}, 1, j)}^{*}(\bar{x})\right| / B_{h(\bar{n}, 1, j), f(\bar{n}, 1, j), \bar{n}, 1}$ increases and we again can get $\bar{b}(\bar{n}, 1, j)$ small. (hence $N$ depends on $M^{\prime}$ and $\left.Q\right)$ and hence it depends also on $P$ (see Construction $C)$ ).

Analogously we can proceed to get the relation which corresponds to (18.3) of theorem 4 of subsection 2.1 of [2], which now will have the following form, for $1 \leq n \leq \bar{n}-1$ : for $2 \leq k \leq Q_{n}+1$ it will be $\|\left[\left(\sum_{j=k+1}^{Q_{n}+1} \sum_{i=1}^{4} \bar{b}(n, j, i) \frac{w_{h(n, j, i), f(n, j, i)}^{*}(\bar{x})}{A_{h(n, j, i), f(n, j, i), n, k}}+\sum_{l=n+1}^{\bar{n}-1} \sum_{j=1}^{Q_{l}+1} \sum_{i=1}^{4} \bar{b}(l\right.\right.$, $\left.j, i) \frac{w_{h(l, j, i), f(l, j, i)}^{*}(\bar{x})}{A_{h(l, j, i), f(l, j, i), n, k}}+\sum_{i=1}^{4} \bar{b}(\bar{n}, 1, i) \cdot \frac{w_{h(\bar{n}, 1, i), f(\bar{n}, 1, i)}^{*}(\bar{x})}{A_{h(\bar{n}, 1, i), f(\bar{n}, 1, i), n, k}}\right) e_{n, k-1} \quad+$ $\left.\sum_{i=1}^{4} \bar{b}(n, k, i) w_{h(n, k, i), f(n, k, i)}^{*}(\bar{x}) . . \bar{w}_{h(n, k, i), f(n, k, i)}\right]-\bar{a}_{n, k} e_{n, k-1 \|<\frac{1}{2^{(M+1) Q}}}$ while, for $\mathrm{k}=1, \quad \|\left[\left(\sum_{j=2}^{Q_{n}+1} \sum_{i=1}^{4} \bar{b}(n, j, i) \frac{w_{h(n, j, i), f(n, j, i)}^{*}(\bar{x})}{A_{h(n, j, i) e, f(n, j, i), n, 1}}+\right.\right.$ $\sum_{l=n+1}^{\bar{n}-1} \sum_{j=1}^{Q_{l}+1} \sum_{i=1}^{4} \bar{b}(l, j, i) \frac{w_{h(l, j, i), f(l, j, i)}^{*}(\bar{x})}{A_{h(l, j, i), f(l, j, i), n, 1}}+\sum_{i=1}^{4} \bar{b}(\bar{n}, 1, i)$ $\left.\left.\frac{w_{h(\bar{n}, 1, i), f(\bar{n}, 1, i)}^{*}(\bar{x})}{A_{h(\bar{n}, 1, i), f(\bar{n}, 1, i), n, 1}}\right) u_{n}+\sum_{i=1}^{4} \bar{b}(n, 1, i) w_{h(n, 1, i), f(n, 1, i)}^{*}(\bar{x}) \cdot \bar{w}_{h(n, 1, i), f(n, 1, i)}\right]-$ $\bar{a}_{n, 1} u_{n} \|<\frac{1}{2^{(M+1) Q}}$. Hence it is possible to verify also (3.1); which completes the proof of theorem 3 .

OBSERVATION 1. Therefore whereas in the real case, for the construction of the approximation $\bar{w}=\sum_{n=1}^{\bar{n}} \bar{b}_{n} w_{f(n)}$ of the element $\bar{u}=\sum_{n=1}^{\bar{n}} \bar{a}_{n} u_{n}$, at each step we consumed only one element $w_{f(n)}$ (see Observation 1 of subsection 2.1), now at each step we need to have at disposal at least four elements $w_{f(n)}$ (even if actually we will use only two of them).

Obviously our choice of the large numbers $H_{n}$ is not necessary, smaller numbers would be also sufficient. The only reason has been of convenience for the applications, indeed by (3.2) of previous theorem 
we used only the elements of $\left(\left(w_{n, k}, w_{n, k}^{*}\right)_{k=1}^{H_{n}+1}\right)_{n=\bar{n}}^{P^{\prime}}$ instead of the whole $\left(\left(w_{n, k}, w_{n, k}^{*}\right)_{k=1}^{H_{n}+1}\right)_{n=1}^{P^{\prime}}$ (see the end of Observation 1 of subsection 2.1).

Remark 4 (COMPLEX MODIFIED GENERATING BIORTHOGONAL SYSTEM (CMGBS))

In theorem 3 we can replace $\left(w_{n}, w_{n}^{*}\right)_{n=1}^{P}$ by means of $\left(v_{n}, v_{n}^{*}\right)_{n=1}^{P}$ where, for each $n$ with $1 \leq n \leq P / 5$ (we can suppose $P$ multiple of 5 ),

$$
\begin{gathered}
v_{5 n-4}=\left(-w_{5 n-4} / D\right)+w_{5 n-3}+e^{i \frac{\pi}{2}} w_{5 n-2}+e^{i \pi} w_{5 n-1}+e^{i \frac{3 \pi}{2}} w_{5 n}, \\
v_{5 n-4}^{*}=\left(-D w_{5 n-4}^{*}\right)+w_{5 n-3}^{*}+w_{5 n-2}^{*}+w_{5 n-1}^{*}+w_{5 n}^{*} \\
\left(\text { indeed } v_{5 n-4}^{*}\left(v_{5 n-4}\right)=(+1)+\left(1+e^{i \frac{\pi}{2}}+e^{i \pi}+e^{i \frac{3 \pi}{2}}\right)=1\right), \\
v_{5 n-3}=w_{5 n-3}+w_{5 n-4} / D, v_{5 n-3}^{*}=w_{5 n-3}^{*}-v_{5 n-4}^{*} \\
=D w_{5 n-4}^{*}-\left(w_{5 n-2}^{*}+w_{5 n-1}^{*}+w_{5 n}^{*}\right), \\
v_{5 n-2}=w_{5 n-2}+w_{5 n-4} / D, v_{5 n-2}^{*}=w_{5 n-2}^{*}-e^{i \frac{\pi}{2}} v_{5 n-4}^{*}= \\
e^{i \frac{\pi}{2}} D w_{5 n-4}^{*}+\left(1-e^{i \frac{\pi}{2}}\right) w_{5 n-2}^{*}-e^{i \frac{\pi}{2}}\left(w_{5 n-3}^{*}+w_{5 n-1}^{*}+w_{5 n}^{*}\right), \\
v_{5 n-1}=w_{5 n-1}+w_{5 n-4} / D, v_{5 n-1}^{*}=w_{5 n-1}^{*}-e^{i \pi} v_{5 n-4}^{*}= \\
e^{i \frac{3 \pi}{2}} D w_{5 n-4}^{*}+\left(1-e^{i \pi}\right) w_{5 n-1}^{*}-e^{i \pi}\left(w_{5 n-3}^{*}+w_{5 n-2}^{*}+w_{5 n}^{*}\right), \\
v_{5 n}=w_{5 n}+w_{5 n-4} / D, v_{5 n}^{*}=w_{5 n}^{*}-e^{i \frac{3 \pi}{2}} v_{5 n-4}^{*}= \\
e^{i \frac{3 \pi}{2}} D w_{5 n-4}^{*}+\left(1-e^{i \frac{3 \pi}{2}}\right) w_{5 n}^{*}-e^{i \frac{3 \pi}{2}}\left(w_{5 n-3}^{*}+w_{5 n-2}^{*}+w_{5 n-1}^{*}\right) .
\end{gathered}
$$

where $D$ is an integer $\geq 2^{\sum_{n=1}^{P}\left\|w_{n}^{*}\right\|}$.

Then the whole statement of theorem 3 continues to hold; we have only to read $\left(v_{n}, v_{n}^{*}\right)_{n=1}^{P}$ instead of $\left(w_{n}, w_{n}^{*}\right)_{n=1}^{P}$.

OBSERVATION 2. Analogously to the Observation 2 of subsection 2.1, suppose that

$$
\begin{array}{r}
\left({ }_{5 n-i}\right)_{i=0}^{4} \subset\left(\left(((h(n, k, i), f(n, k, i)))_{i=1}^{4}\right)_{k=1}^{H_{n}+1}\right)_{n=1}^{\bar{n}-1} \cup((h(\bar{n}, 1, i), \\
f(\bar{n}, 1, i)))_{i=1}^{4}
\end{array}
$$


of thesis of previous theorem, then $\left|w_{5 n-4}^{*}(\bar{x})\right|$ is much larger than $\sum_{i=0}^{3}\left|w_{5 n-i}^{*}(\bar{x})\right|$ and $D$ is much larger than $\left|w_{5 n-4}^{*}(\bar{x})\right|$; therefore we can approximate $\left(v_{5 n-i}^{*}(\bar{x}) v_{5 n-i}\right)_{i=0}^{3}$ by means of

$$
\begin{gathered}
\left(D w_{5 n-4}^{*}(\bar{x}) w_{5 n-3}, e^{i \frac{\pi}{2}} D w_{5 n-4}^{*}(\bar{x}) w_{5 n-2},\right. \\
\left.e^{i \pi} D w_{5 n-2}^{*}(\bar{x}) w_{5 n-1}, e^{i \frac{3 \pi}{2}} D w_{5 n-4}^{*}(\bar{x}) w_{5 n}\right) ;
\end{gathered}
$$

that is we have the same coefficient but with four possibilities for the angles, which is what we need.

\section{ON THE EXISTENCE OF THE UCP-BASIS ALSO IN THE COMPLEX CASE}

\subsection{The direct extension to the complex field}

OBSERVATION 1. On the Walsh matrix in the complex field.

Let $\left(o_{n}\right)_{n=1}^{2^{S}}$ be the natural basis of $E=l_{\infty}^{2^{S}}$ with $\left(o_{n}, o_{n}^{*}\right)_{n=1}^{2^{S}}$ biorthogonal. Let $\left(\widehat{o}_{n}, \widehat{o}_{n}^{*}\right)_{n=1}^{2^{S}}$ be the biorthogonal system derived from $\left(o_{n}, o_{n}^{*}\right)_{n=1}^{2^{S}}$ by means of the matrix of Walsh.

Not all the properties of the Walsh matrix keep exactly in the complex field. For instance it is obvious, that $\widehat{o}_{1} \cup\left(\widehat{o}_{2^{m-1}+1}\right)_{m=2}^{S}$ is 1 -equivalent to the natural basis of $l_{1}^{S}$. But this fact does not hold in the complex case; indeed if $\left(e_{1}^{\prime}, e_{2}^{\prime}\right)$ is the natural basis of $l_{\infty}^{2}$, the application of the Walsh matrix gives $\left(e_{1}, e_{2}\right)$, with $e_{1}=e_{1}^{\prime}+e_{2}^{\prime}$ and $e_{2}=e_{1}^{\prime}-e_{2}^{\prime}$ and in the real case $\left(e_{1}, e_{2}\right)$ is the natural basis of $l_{1}^{2}$; while in the complex case we have for instance that

||$e_{1}+e^{i \frac{\pi}{2}} e_{2} \|=||\left(e_{1}^{\prime}+e_{2}^{\prime}\right)+e^{i \frac{\pi}{2}}\left(e_{1}^{\prime}-e_{2}^{\prime}\right)||=\max \left(\left|1+e^{i \frac{\pi}{2}}\right|,\left|1-e^{i \frac{\pi}{2}}\right|\right)=\sqrt{2}$.

Obviously by means of little generalizations of the Walsh matrix we can always have sequences $(1+\varepsilon)$-equivalent to the natural basis of $l_{1}^{N}$ for each positive integer $N$ and for any $\varepsilon>0$. For instance if $\left(o_{n}\right)_{n=1}^{N^{N+1}}$ is the natural basis of $l_{\infty}^{N^{N+1}}$ in the complex field, setting

$$
e_{1}=\sum_{k=1}^{N^{N}} o_{1, k} \text { with } o_{1, k}=\sum_{l=(k-1) N+1}^{k N} e^{i \frac{l-(k-1) N}{N} 2 \pi} o_{l} \text { for } 1 \leq k \leq N^{N}
$$


(hence

$$
\begin{gathered}
\left.o_{1,1}=\sum_{l=1}^{N} e^{i\left(\left(\frac{l}{N}\right) 2 \pi\right)} o_{l}, . ., o_{1, N^{N}}=\sum_{l=\left(N^{N}-1\right) N+1}^{N^{N}} e^{\left.i\left(\frac{l-\left(N^{N}-1\right) N}{N}\right) 2 \pi\right)} o_{l}\right), \\
e_{2}=\sum_{k=1}^{N^{N-1}} o_{2, k} \text { with } o_{2, k}=\sum_{l=(k-1) N+1}^{k N} e^{i \frac{l-(k-1) N}{N} 2 \pi} o_{1, l} \text { for } 1 \leq k \leq N^{N-1}
\end{gathered}
$$

and so on till

$$
e_{N}=\sum_{k=1}^{N} o_{N, k} \text { with } o_{N, k}=\sum_{l=(k-1) N+1}^{k N} e^{i \frac{l-(k-1) N}{N} 2 \pi} o_{N-1, l} \text { for } 1 \leq k \leq N \text {; }
$$

we immediately have that, for any $\left(a_{n}\right)_{n=1}^{N}$ of complex numbers,

$$
\begin{aligned}
& \left|\left\|\sum_{n=1}^{N} a_{n} e_{n}\right\|-\sum_{n=1}^{N}\right| a_{n}|| \leq \varepsilon(N) \sum_{n=1}^{N}\left|a_{n}\right| \text { for } \varepsilon(N)=\left|1-e^{i \frac{2 \pi}{N}}\right| \\
& \quad=\sqrt{2} \sqrt{1-\cos \frac{2 \pi}{N}},
\end{aligned}
$$

that is $\left(e_{n}\right)_{n=1}^{N}$ is $(1+\varepsilon(N))$-equivalent to the natural basis of $l_{1}^{N}$ in the complex field.

However, for what concerns the extension of the UCP-basis in the complex field, these generalizations of the Walsh matrix are not necessary.

By means of the same ideas of the generalization to the complex case of the generating biorthogonal systems of section 2, it is possible to generalize to the complex case all the theorems and lemma of sections $3 . .6$ of [2]; in particular we point out that the proofs of sections 6 of [2] work also in the complex case). But only apart from lemma 9 of subsection 2.3 of [2], for which the extension to the complex case is still open. Let us recall this simple lemma. 
We say that, given a sequence $\left(a_{k}\right)_{k=1}^{P}$ of numbers, $\left(\sum_{k=1}^{K} a_{k}\right)_{K=1}^{P}$ is $(M, \varepsilon)$-monotone if, for each integers $K$ and $Q$ with $1 \leq K \leq Q \leq P,\left|\sum_{k=1}^{K} a_{k}\right| \leq\left|\sum_{k=1}^{Q} a_{k}\right|+M^{\prime} \cdot \max \left(\left|a_{k}\right|: 1 \leq k \leq P\right)+\varepsilon$.

Lemma 5 Let $\left(e_{n}^{\prime}\right)_{n=1}^{2^{Q}}$ be the natural basis of $l_{1}^{2 Q}$ and set $e_{1}=e_{1}^{\prime}$ and $e_{n}=e_{n}^{\prime}-e_{n-1}^{\prime}$ for $2 \leq n \leq 2^{Q}$, then for each $\left(a_{n}\right)_{n=1}^{2^{Q}}$ of numbers there exists a permutation $(\pi(n))_{n=1}^{2 Q}$ of $(n)_{n=1}^{2^{Q}}$ such that:

$$
\begin{gathered}
\text { (i) }\left(\left|\sum_{n=1}^{q} a_{\pi(n)}\right|\right)_{q=1}^{2^{Q}} \text { is }(1,0)-\text { monotone; } \\
\text { (ii) }\left(\left\|\sum_{n=1}^{q} a_{\pi(n)} e_{\pi(n)}\right\|\right)_{q=1}^{2^{Q}} \text { is }(0,0)-\text { monotone. }
\end{gathered}
$$

Therefore, if it would be possible to extend to the complex case the previous lemma, it would automatically follow the extension of the UCP-basis in the complex field; indeed it would be necessary only some obvious modifications in the construction of the UCP-basis in the real field. Moreover also for all the theorems and lemmas of section 5 only reasonable modifications would be necessary.

I only point out that, for instance, in the particular case of $\left|a_{n}\right|=$ $a>0$ for $1 \leq n \leq 2^{Q}$, the extension of previous lemma to the complex case is easy.

\subsection{Passage through the real associated space}

Let $X$ be a complex separable Banach space.

I recall that $\left(w_{n}\right)$ with $\left(w_{n}, w_{n}^{*}\right)$ biorthogonal is an $M^{\prime}$-basis for $X$ if the closure of $\operatorname{span}\left(w_{n}\right)$ is $X$ and if, for any $x$ of $X$, the condition $w_{n}^{*}(x)=0$ for every $n$ implies that $x=O$ (the null vector).

Then let $\left(w_{n}\right)$ be any $M^{\prime}$-basis for $X$ with $\left(w_{n}, w_{n}^{*}\right)$ biorthogonal, we pass to another sequence $\left(u_{n}\right)$ with $u_{2 n-1}=w_{n}$ and $u_{2 n}=i w_{n}$ for 
each $n$; let us call $X_{r}$ the closure of $\operatorname{span}\left(u_{n}\right)$ in the field of the real numbers.

Then if $x \in X$ there exists $\left(\left(c_{p, n}\right)_{n=1}^{q(p)}\right)_{p=1}^{\infty}$ of complex numbers, with $c_{p, n}=c_{p, n}^{\prime}+i c_{p, n}^{\prime \prime}$ for each $n$ and $p$, such that $\left\|x-\sum_{n=1}^{q(p)} c_{p, n} w_{n}\right\|<$ $\frac{1}{p}$ for each $p$; hence we have that $\left\|x-\sum_{n=1}^{q(p)}\left(c_{p, n}^{\prime} u_{2 n-1}+c_{p, n}^{\prime \prime} u_{2 n}\right)\right\|<\frac{1}{p}$ for each $p$ hence $X \subseteq X_{r}$. On the other hand let $y \in X_{r}$, there exists $\left(\left(c_{p, n}^{\prime}, c_{p, n}^{\prime \prime}\right)_{n=1}^{q(p)}\right)_{p=1}^{\infty}$ of real numbers such that $\| y-\sum_{n=1}^{q(p)}\left(c_{p, n}^{\prime} u_{2 n-1}+\right.$ $\left.c_{p, n}^{\prime \prime} u_{2 n-1}\right)\|=\| y-\sum_{n=1}^{q(p)}\left(c_{p, n}^{\prime}+i c_{p, n}^{\prime \prime}\right) w_{n} \|<\frac{1}{p}$ for each $p$ hence $X \supseteq X_{r}$; that is the two sets $X$ and $X_{r}$ coincide.

Definition. Let $\left(u_{n}\right)$ be UCP-basis for $X_{r}$, we say that $\left(u_{n}\right)$ has property $\mathrm{P}$ if, for each $x \in X_{r}$ with $x=\sum_{n=1}^{\infty} a_{n} u_{\pi(n)}$ for some permutation $(\pi(n))$ of $(n)$ (hence $\left(a_{n}\right)$ is unique), $(\pi(n))_{n=1}^{\infty}$ is always a permutation of the couples $\left(u_{2 n-1}, u_{2 n}\right)$ for each $n$, that is these couples are armoured hence there exists another permutation $(\tilde{\pi}(n))$ of $(n)$ such that $\left(u_{\pi(2 n-1)}, u_{\pi(2 n)}\right)=\left(u_{2 \widetilde{\pi}(n)-1}, u_{2 \widetilde{\pi}(n)}\right)$ for each $n$.

Obviously $\left(u_{n}\right)$ is not linearly independent in $X$, however it is easy to recognize that

" $\left(w_{n}\right)$ is $M^{\prime}$-basis for $X \Longleftrightarrow\left(u_{n}\right)$ is $M^{\prime}$-basis for $X_{r} "$;

" $\left(w_{n}\right)$ is Schauder basis for $X \Longleftrightarrow\left(u_{n}\right)$ is Schauder basis for $X_{r} "$;

" $\left(w_{n}\right)$ is UCP-basis for $X \Longleftrightarrow\left(u_{n}\right)$ is UCP-basis for $X_{r}$ with property $P^{\prime \prime}$ (indeed if $\left(u_{n}\right)$ has property $P$, it is also

$$
\begin{gathered}
x=\sum_{n=1}^{\infty} a_{n} u_{\pi(n)}=\sum_{n=1}^{\infty}\left(a_{2 n-1} u_{\pi(2 n-1)}+a_{2 n} u_{\pi(2 n)}\right)=\sum_{n=1}^{\infty}\left(a_{2 n-1} u_{2 \widetilde{\pi}(n)-1)}\right. \\
\left.+a_{2 n} u_{2 \widetilde{\pi}(n)}\right)=\sum_{n=1}^{\infty}\left(a_{2 n-1}+i a_{2 n}\right) w_{\widetilde{\pi}(n)}=\sum_{n=1}^{\infty} c_{n} w_{\widetilde{\pi}(n)}
\end{gathered}
$$

with $c_{n}=a_{2 n-1}+i a_{2 n}$ for each $n$ hence also $\left(c_{n}\right)$ is unique). 


\section{RIFERIMENTI BIBILIOGRAFICI}

[1] ENFLO,P. A counterexample to the approximation problem in Banach spaces. Acta Mathematica 130 (1973), 309-317.

[2] TERENZI,P. Every separable Banach space has a basis with uniformly controlled permutations. Dissertationes Mathematicae 439 (2006), 1-177. 